\title{
IMPLEMENTATION WITH EX POST HIDDEN ACTIONS
}

\author{
Caleb M. Koch \\ ETH Zurich, Switzerland \\ caleb.m.koch@gmail.com
}

\begin{abstract}
We study implementation in settings where agents take strategic actions that influence preferences over mechanism outcomes and yet are hidden from the mechanism designer. We show that such settings can arise in entry auctions for markets, and that the Vickery-Clarke-Groves mechanism is not necessarily truthful. In this paper we first formalize so-called ex post hidden actions, we then characterize social choice functions that can be implemented in a way that is robust with respect to ex post hidden actions, and finally we propose a mechanism to do so. The model allows agents to have multi-dimensional types and to have quasi-linear utilities in money. We showcase these results by identifying social choice functions that can and cannot be implemented in entry auctions for Cournot competition models.
\end{abstract}

Keywords: Ex post hidden actions, dominant-strategy incentive compatibility, auctions.

JEL Classification Numbers: C72, D70, D82.

I am indebted to Heinrich Nax for his advice and encouragement. This paper is based on my PhD thesis. I am grateful to Rabah Amir, Yen-Lin Chiu, Stefano Duca, Dirk Helbing, Osama Khan, Bary Pradelski, John Quah, Alain Rossier, Niccolò Urbinati, participants of the Conference on Economic Design '19 at the University of Corvinus, as well as an excellent editor and three anonymous referees for helpful comments. Financial support from the European Commission through the ERC Advanced Investigator Grant 'Momentum' (Grant 324247) is gratefully acknowledged. The paper was previously distributed under the title, "Implementation with secrets" and "Implementation with strategic private information". Any remaining errors are my own. 


\section{INTRODUCTION}

$\mathbf{M}$ ECHANISM design, sometimes called "reverse game theory", is the theory of designing rules and regulations so that self-interested agents can achieve social goals. A typical mechanism design problem is formulated as follows. First, each agent has private information. Second, each agent may submit a report to the mechanism designer about his/her private information (this report may or may not be truthful). Finally, the mechanism designer makes a social decision based on agents' reports. This typical setup, however, assumes that the only strategic action that agents take is sending the mechanism designer a report. As such, mechanism design assumes that agents cannot influence other agents' payoffs via strategic interactions outside the mechanism, and any strategic actions other than reporting are thus irrelevant for the design of a mechanism itself. In other words, mechanisms are typically assumed to be a closed system.

However, there are economic settings that might challenge the closed system assumption. For example, consider a spectrum auction where firms compete for (scarce) spectrum licenses to enter into the telecommunications market (McAfee \& McMillan, 1996; Milgrom, 2000; Klemperer, 2002a,b). What is the timing of such an auction? First, firms make bids for spectrum licenses. Second, firms receive licenses based on all firms' bids (and pay the government according to the auction payment scheme). Third - and perhaps most importantly - firms with spectrum licenses compete for market share. This third point is relevant because it means that firms can influence each others' payoffs via strategic market actions that are beyond the spectrum auction. However, the telecommunications market has, to the best of the author's knowledge, never been modeled explicitly when designing spectrum auctions.

Do strategic market actions after auctions make a difference for mechanism design? We show that the answer is yes: in the main text (see, e.g. Example 4 and Remark 4), we give an example to illustrate where the Vickery-ClarkeGroves Mechanism is not strategy-proof or truthful when strategic interactions after a mechanism are modeled explicitly, even if there exists a unique Nash equilibrium. More generally, we attempt to clarify how and why ex post hidden actions after a mechanism can play an important role in influencing mechanisms that are designed to implement social goals.

In this paper, we address these questions by studying environments in which Journal of Mechanism and Institution Design 5(1), 2020 
agents can take hidden actions from the mechanism designer after mechanism outcomes. Such hidden strategic actions can play a crucial role in determining personal and/or others' preferences over social decisions. To reiterate, strategic actions differ from exogenous private information as they can (i) be selected strategically and (ii) may be subject to change after agents give information to the mechanism designer. For example, hidden actions could represent firms' market decisions after spectrum licenses are distributed, where such market decisions are clearly unknown to the mechanism designer before licenses are distributed.

This paper makes two major contributions. The first is formalizing so-called 'ex post hidden actions'. As noted above, an ex post hidden action is taken by an agent after a mechanism outcome, is unknown to a mechanism designer, and yet can be crucial for determining personal and/or others' preferences over mechanism outcomes. An ex post hidden action may include pricing decisions, merger and acquisition decisions, contract negotiations, etc.

The second contribution is characterizing social choice functions that can be implemented in a way that is robust to ex post hidden actions. Concretely, the class of mechanisms considered here proceeds as follows. First, agents report preferences over mechanism outcomes (this can be thought of reporting a utility function); to reiterate, agents do not report ex post hidden actions. Second, the mechanism designer makes a social decision based on agents' reports, and agents pay the mechanism designer based on a pre-specified payment scheme. Finally, after the social decision, agents take their (ex post) hidden actions and receive their payoffs.

Such mechanisms can be viewed as a strategic counterpart to robust mechanism design (Bergemann \& Morris, 2005). A robust mechanism asks each agent to report private information but not his/her belief, which is also private information. Such mechanisms are thus robust to unreported exogenous private information. Here, mechanisms can be view as being robust with respect to unreported strategic private information. We comment on this further in the related literature subsection below.

The main theorems in this paper characterize social choice functions that can be implemented in dominant-strategies when agents take ex post hidden actions. The characterization boils down to a single 'monotonicity' condition that must be satisfied by the social choice function. This condition requires two properties: $(i)$ the social choice function gives agents with 'higher types' priority to 'better outcomes', and (ii) matching higher types with better 
outcomes exhibits increasing returns. Implementation is generally impossible without both conditions.

We show that implementation with ex post hidden actions is indeed possible and practical by studying entry mechanisms for a Cournot market (which builds on an example in Dasgupta \& Maskin, 2000). In particular, firms bid to enter into a Cournot market, and firms' valuations of mechanism outcomes depend on ex post hidden actions because of strategic interactions in the Cournot market. It turns out that our characterization can delineate between social choice functions that can and cannot be implemented in a way that is robust to the Cournot market. We show that: $(i)$ there exists a truthful mechanism that can maximize consumer surplus and that, (ii) even if there is a unique Nash equilibrium in the Cournot market, there are no truthful mechanisms that can maximize producer surplus (at Nash equilibrium), including the Vickery-Clarke-Groves Mechanism.

This paper draws primarily from two research streams in mechanism design.

The first research stream from which this paper draws is robust mechanism design (Bergemann \& Morris, 2005). The fundamental goal of robust mechanism design is to identify conditions under which a mechanism can elicit information truthfully without asking for beliefs. A belief is modeled as exogenous private information, which means that a robust mechanism can be viewed as being robust with to exogenous private information. This literature has identified various necessary and sufficient conditions under which agents report truthfully regardless of privately held beliefs (see, e.g., Bergemann \& Morris, 2011, and Bergemann et al., 2011).

This paper tries to offer a strategic counterpart to the current robust mechanism design literature: rather than beliefs, the main results relate to when one can design mechanisms where agents report truthfully regardless of ex post hidden actions. This opens up several possibilities for robust mechanism design, such as allowing beliefs to be influenced by cheap talk.

The second research stream focuses on incentive compatibility in quasilinear environments with exogenous private information. Ex post hidden actions in a mechanism design environment are closely related to studying environments with interdependent preferences. Jehiel \& Moldovanu (2001) show that the Bayesian incentive compatibility of the efficient allocation rule is generally impossible. We use an example that is closely related to the ex post hidden action context (when the Nash equilibirum is unique, preferences with 
ex post hidden action reduce to interdependent preferences). ${ }^{1}$ This paper is closer to the strand of literature that focuses on dominant-strategy incentive compatibility - which effectively requires Bayesian implementation within every information structure (see, e.g. Dasgupta et al., 1979) - as dominantstrategies are the focus here. The advantage of the dominant-strategy vs. Bayesian approach is its 'detail free' nature (Wilson, 1987), in the sense that a designer does not need information about agents' priors in order to formulate expected profits. $^{2}$

The most closely related papers from this literature focus on deterministic mechanisms and explore necessary and sufficient conditions for implementation. Roberts (1979) was the first to do so in settings where agents' types come from an unrestricted type domain. The main finding of Roberts is a necessary and sufficient condition, called "Positive Association Differences" (PAD), that characterizes dominant-strategy incentive compatibility on such domains. The drawback of PAD is its inapplicability in many relevant economic settings, such as the allocation of private goods, since it precludes assumptions such as free disposal and externality in consumption. ${ }^{3}$ Bikhchandani et al. (2006) resolved these issues and characterized dominant-strategy implementation on a restricted type domain that admits assumptions such as free disposal. The advantage of the approach taken in Bikhchandani et al. (2006) is its applicability to a large class of economically relevant settings, including auctions.

The rest of the paper proceeds as follows. In Section 2, we introduce the model. In Section 3, we present the 'implementation with ex post hidden actions' problem statement. In Section 4, we present our main results. We conclude in Section 5. All proofs, unless otherwise provided, are relegated to the appendices.

1 For other references from the Bayesian incentive compatibility literature that are relevant to this paper, see Myerson (1981), Rochet (1987), McAfee \& McMillan (1988), Jehiel et al. (1999), Williams (1999), Krishna \& Maenner (2001), and Milgrom \& Segal (2002).

2 Relatedly, Chung \& Ely (2007) provide a theoretical foundation for dominant-strategy implementation by proving that such mechanisms are maximin optimal.

3 See Bikhchandani et al. (2006) for an extended discussion on the limitations of using Positive Association Differences in applied economic models. 


\section{THE MODEL}

This section presents the model underlying implementation with ex post hidden actions. Definitions are in bold.

\subsection{The Environment}

Consider a finite set of agents $\mathcal{N}=\{1,2, \ldots, n\}$ and mechanism outcomes $\boldsymbol{Q}=\left\{q_{1}, q_{2}, \ldots, q_{K}\right\}$. Each agent $i \in \mathcal{N}$ has private information $V_{i} \in \mathcal{D}_{i}$ and takes an ex post hidden action $s_{i} \in \mathcal{S}_{i}$ (where $\mathcal{S}_{i}$ may be finite or infinite). A collection of ex post hidden actions is denoted as $s=\left(s_{1}, \ldots, s_{n}\right) \in$ $\times_{j \in \mathcal{N}} \mathcal{S}_{j}=: \mathcal{S}$. Private information and hidden actions determine an agent's utility of an outcome $q \in Q$ and money $m \in \mathbb{R}$, which is represented as $\Pi_{i}\left(\cdot, s ; V_{i}\right): Q \times \mathbb{R} \rightarrow \mathbb{R}:$

$$
\Pi_{i}\left(q, m, s ; V_{i}\right)=U_{i}\left(q, s ; V_{i}\right)+m .
$$

It is useful to let $U_{i}\left(q, s ; V_{i}\right) \equiv V_{i}(q, s)$ and refer to $V_{i}$ as agent $i$ 's type. We refer to $\mathcal{D}_{i} \subseteq\left\{V_{i}: Q \times \mathcal{S} \rightarrow \mathbb{R}\right\}=: \mathscr{D}_{i}$ as the domain of possible types and let $\mathcal{D}:=\times_{j} \mathcal{D}_{j}$.

Definition 1 (Social Choice Environment). An environment with ex post hidden actions is defined by a tuple $\left\{\mathcal{N},\left(\mathcal{S}_{j}, \mathcal{D}_{j}, V_{j}\right)_{j \in \mathcal{N}}, Q\right\}$.

By fixing an outcome $q \in Q$, an environment with ex post hidden actions can be reduced to a non-cooperative game, $\left\{\mathcal{N},\left(\mathcal{S}_{j}, V_{j}(q, \cdot)\right)_{j \in \mathcal{N}}\right\}$, where $\mathcal{S}_{j}$ is agent $j$ 's action space.

From a standard mechanism design perspective, one could view agents as having a mix of independent and (strategically) interdependent types. On the one hand, agents have independent types insofar as $V_{i}(\cdot)$ does not depend on $V_{-i}$. On the other hand, agents have (strategically) interdependent types insofar as $V_{i}(\cdot)$ depends on $s_{-i} \in \mathcal{S}_{-i}$.

We give an example of an environment with ex post hidden actions below.

Example 1 (Oil Drilling; Dasgupta \& Maskin, 2000). A mechanism designer is distributing two licenses to drill oil. Each (prospective) firm's valuation of a license equals its expected profit in the oil market. As such, the challenge for the mechanism designer is that each firm decides its strategic actions in the market after licenses are distributed. This means that the mechanism designer cannot distribute licenses based on firms' oil drilling plans. 
To model this more formally, let $\mathcal{N}=\{1,2,3\}$ denote the set of prospective firms. $Q=\{(1,1,0),(1,0,1),(0,1,1)\}$ denotes the set of possible license distributions ( 1 represents a firm receiving a license, 0 represents a firm not receiving a license). Let $s_{i} \in \mathcal{S}_{i}=\left[0, S_{i}\right]$ denote firm $i$ 's oil production, which is an ex post hidden action. A firm's marginal cost of production, $V_{i} \in \mathcal{D}_{i}=\left[0, D_{i}\right]$, is exogenous private information. We model a firm's profit in the oil market as a Cournot competition. This means that firm $i$ 's utility is given as

$$
\mathcal{U}_{i}\left(q, m, s ; V_{i}\right):=m+ \begin{cases}\overbrace{s_{i} \cdot P(q \cdot s)-s_{i} V_{i}}^{\text {Profit from oil market }} & \text { if } q_{i}=1 \\ \underbrace{0}_{\text {Profit }=0 \text { without license }} & \text { otherwise }\end{cases}
$$

where $P: \mathbb{R}_{+} \rightarrow \mathbb{R}_{+}$is the inverse demand function.

In this example, a firm's oil pumping decision in the market is an ex post hidden action because pumping oil: (i) impact a firm's preferences over mechanism outcomes and (ii) takes shape after the mechanism outcome. The setup above forms the basis of examples throughout the paper.

\subsection{Social Choice Mechanism}

An agent's ex post hidden action takes on a different role than the standard concept of private information. In general, an agent may choose $s_{i} \in \mathcal{S}_{i}$ based on others' types, others' strategic actions, and is determined after the mechanism outcome. This means that the mechanism designer cannot elicit ex post hidden actions from agents, as such information is subject to change. Instead, the designer must solely rely on exogenous private information to make social decisions.

As such, we consider social choice mechanisms that require each agent to report exogenous private information, but not ex post hidden actions. We thus define a social choice function $f: \mathcal{D} \rightarrow Q$ as a mapping from agents' reported types to an outcome. A payment function $p=\left(p_{1}, p_{2}, \ldots, p_{n}\right): \mathcal{D} \rightarrow \mathbb{R}^{n}$ maps agents' reported types to a money payment for each agent. Finally, a social choice mechanism is denoted by a pair $(f, p)$. 


\subsection{Timing}

Having spelled out the model ingredients, it is worth noting the exact timing of the mechanisms that will be considered. (Other timings are perhaps possible, but we restrict attention here for the sake of discussion.)

1. Each agent reports a type $\tilde{V}_{i} \in \mathcal{D}_{i}$ simultaneously.

2. The mechanism designer makes a social decision based on everybody's report, $f\left(\tilde{V}_{1}, \ldots, \tilde{V}_{n}\right)$.

3. Each agent pays the mechanism designer money based on everybody's reported types, $p\left(\tilde{V}_{1}, \ldots, \tilde{V}_{n}\right)=\left(p_{1}, \ldots, p_{n}\right) \in \mathbb{R}^{n}$.

4. With everybody knowing everybody's true type, each player takes an action, $s=\left(s_{1}, \ldots, s_{n}\right) \in \mathcal{S}$.

5. Finally, each player receives his/her payoff,

$$
V_{i}\left(f\left(\tilde{V}_{1}, \ldots, \tilde{V}_{n}\right), s\right)-p_{i}\left(\tilde{V}_{1}, \ldots, \tilde{V}_{n}\right) .
$$

\section{PROBLEM STATEMENT}

In this section, we formalize the main research question of this paper.

\subsection{Gathering Information Truthfully}

Consider an environment with ex post hidden actions. The mechanism designer's goal is, first, to gather information from agents and, second, to make a social decision based on this information. The challenge is that the mechanism designer cannot make payments based on agents' ex post hidden actions, $s_{i} \in \mathcal{S}_{i}$, but only on agents' exogenous private information, $V_{i} \in \mathcal{D}_{i}$. Informally, our criterion for truthfulness is that each agent has a (weakly) dominant-strategy to report his/her true $V_{i}$ while all agents' strategic actions follow a Nash equilibrium.

We introduce some notation to define our notion of truthfulness formally. As noted above, any outcome $q \in Q$ reduces an environment with ex post hidden actions to a non-cooperative game, $\left\{\mathcal{N},\left(\mathcal{S}_{j}, V_{j}(q, \cdot)\right)_{j \in \mathcal{N}}\right\}$. Let $\mathcal{N} \mathcal{E}^{\left(V_{i}, V_{-i}\right)}(q) \subseteq$ $\mathcal{S}$ denote the set of Nash equilibria of the non-cooperative game under outcome 
$q \in Q$ and with types $\left(V_{i}, V_{-i}\right)$. When working with a social choice function $f$, it is often useful to let $\mathcal{N} \mathcal{E}^{\left(V_{i}, V_{-i}\right)}\left(f\left(V_{i}, V_{-i}\right)\right) \equiv \mathcal{N} \mathcal{E}^{\left(V_{i}, V_{-i}\right)}\left(V_{i}, V_{-i}\right)$.

All results in the following paper build on the following assumption, which states that there exists at least one Nash equilibrium at every combination of types $V \in \mathcal{D}$ and possible mechanism outcomes $q \in Q$. Why is this assumption important? Among other reasons, it gives players credible deviations from telling the truth - otherwise the mechanism designer is not worried about non-truthful strategies because it leads to non-Nash outcomes. Relatedly, the absence of Nash equilibria renders it impossible to define a payment function, which necessarily relies on Nash outcomes to provide a foundation on which a payment function can be built.

Assumption 1 An environment with ex post hidden actions satisfies regularity if $\mathcal{N} \mathcal{E}^{V}(q)$ is non-empty for all $V \in \mathcal{D}$ and $q \in Q$.

The following definition formalizes what it means for an agent to always have a truthful dominant-strategy.

Definition 2 (Truthful Dominant-Strategy). For any $(f, p)$ and an agent $i$, truthful reporting is a (weakly) dominant-strategy if, for every $V_{-i} \in \mathcal{D}_{-i}$, it is weakly optimal for any type $V_{i} \in \mathcal{D}_{i}$ to report truthfully, that is,

$$
V_{i}\left(f\left(V_{i}, V_{-i}\right), s^{*}\right)-p_{i}\left(V_{i}, V_{-i}\right) \geq V_{i}\left(f\left(\tilde{V}_{i}, V_{-i}\right), \tilde{s}^{*}\right)-p_{i}\left(\tilde{V}_{i}, V_{-i}\right)
$$

for all untruthful reports $\tilde{V}_{i} \in \mathcal{D}_{i} \backslash\left\{V_{i}\right\}$ that yield different outcomes $\left(f\left(V_{i}, V_{-i}\right) \neq\right.$ $\left.f\left(\tilde{V}_{i}, V_{-i}\right)\right)$, Nash equilibria that result from reporting truthfully, i.e. $s^{*} \in$ $\mathcal{N} \mathcal{E}^{\left(V_{i}, V_{-i}\right)}\left(V_{i}, V_{-i}\right)$, and Nash equilibria that result from mis-reporting, i.e. $\tilde{s}^{*} \in \mathcal{N} \mathcal{E}^{\left(V_{i}, V_{-i}\right)}\left(\tilde{V}_{i}, V_{-i}\right)$.

Notice that a truthful dominant-strategy is defined with respect to the true Nash equilibria of the underlying game (that is, $\left.\mathcal{N} \mathcal{E}^{\left(V_{i}, V_{-i}\right)}(\cdot)\right)$ and not Nash equilibria based on reports (that is, $\mathcal{N} \mathcal{E}^{\left(\tilde{V}_{i}, V_{-i}\right)}(\cdot)$ on the right-hand side of (2)).

Dominant-strategy incentive compatibility extends the concept of a truthful dominant-strategy to all agents.

Definition 3 (Nash Dominant-Strategy Incentive Compatibility). A social choice mechanism $(f, p)$ satisfies Nash dominant-strategy incentive compatibility (DSIC) if truthful reporting is a dominant strategy for all agents. 
In other words, if a social choice mechanism satisfies Nash DSIC, then no agent can strictly gain by misreporting $\left(\tilde{V}_{i}\right)$ to induce a new Nash equilibrium outcome $\left(\tilde{s}^{*}\right)$.

Nash DSIC underlies a so-called "truthful" social choice function.

Definition 4 (Truthful). A social choice function $f$ is truthful if there exists a payment function $p$ such that $(f, p)$ satisfies Nash DSIC; $p$ is said to implement $f$.

The main goal of this paper is to analyze social choice functions that can be implemented in environments with ex post hidden actions.

Before proceeding, we make three remarks about the definition of 'truthful'.

Remark 1. All results in this paper are formulated in terms of (i) reporting $V_{i}$ truthfully as a dominant-strategy and (ii) $s^{*} \in \mathcal{S}$ following a Nash equilibrium of the true underlying game. Concerning the latter, everything can be easily adapted to represent rationalizable strategies rather than Nash equilibria. ${ }^{4}$ All of the proofs would proceed as stated (with a few modifications).

Remark 2. What doss the economic interpretation of 'truthful' mean in light of ex post hidden actions? We have two interpretations in mind. First, it may be that private information is common knowledge (in the sense of Aumann, 1976), and the mechanism designer knows that agents have common knowledge but does not know agents' private information - such a setting might be appropriate for small- $n$ environments, or when agents have interacted in mechanism settings together repeatedly. Second, agents may report while not knowing others' private information, and then types of all agents are revealed after the mechanism outcome - such a setting may be appropriate for, e.g. the oil drilling license auction or some spectrum auction settings. It is our hope that main results and proofs can be adapted to different definitions of truthfulness that may be more appropriate in specific economic settings.

\section{A CHARACTERIZATION OF IMPLEMENTATION WITH EX POST HIDDEN ACTIONS}

This section contains the main results of the paper, which are presented as follows. First, we derive a straightforward condition that is necessary for

4 See Bernheim (1984) and Pearce (1984) for the definition of rationalizable strategies and Bergemann et al. (2011) for a characterization of implementation using rationalizable strategies in robust mechanism design. 
implementation. Second, we show that this condition is also sufficient when we focus on unit-demand auctions with identical goods, and third we use this to study different social choice functions in a Cournot entry market. Finally, we present a generalized characterization of implementation with ex post hidden actions.

\subsection{A Necessary Condition for Implementation}

In implementation theory, truthfulness is often characterized by a 'monotonicity condition' (e.g., 'Maskin monotonicity', Maskin, 1999). Here, we take the same approach.

The next definition introduces a monotonicity condition for social choice functions.

Definition 5 (Nash Monotonicity, Nash-MON). In an environment with ex post hidden actions, a social choice function $f$ satisfies Nash monotonicity (Nash-MON) if, for all $i \in \mathcal{N}, V_{-i} \in \mathcal{D}_{-i}$, and $V_{i}, \tilde{V}_{i} \in \mathcal{D}_{i}$ such that $f\left(V_{i}, V_{-i}\right) \neq$ $f\left(\tilde{V}_{i}, V_{-i}\right)$,

marginal gain for $V_{i}$ to report truthfully vs. report $\tilde{V}_{i}$

$$
\begin{aligned}
& \overbrace{V_{i}\left(f\left(V_{i}, V_{-i}\right), s^{*}\right)-V_{i}\left(f\left(\tilde{V}_{i}, V_{-i}\right), s^{* *}\right)} \\
& \geq \underbrace{\tilde{V}_{i}\left(f\left(V_{i}, V_{-i}\right), \tilde{s}^{* *}\right)-\tilde{V}_{i}\left(f\left(\tilde{V}_{i}, V_{-i}\right), \tilde{s}^{*}\right)}
\end{aligned}
$$

marginal gain for $\tilde{V}_{i}$ to misreport with $V_{i}$ vs. report truthfully

for all Nash equilibria that result from:

- player $V_{i}$ reporting truthfully, i.e. $s^{*} \in \mathcal{N} \mathcal{E}^{\left(V_{i}, V_{-i}\right)}\left(V_{i}, V_{-i}\right)$,

- player $V_{i}$ misreporting, i.e. $s^{* *} \in \mathcal{N} \mathcal{E}^{\left(V_{i}, V_{-i}\right)}\left(\tilde{V}_{i}, V_{-i}\right)$,

- player $\tilde{V}_{i}$ reporting truthfully, i.e. $\tilde{s}^{*} \in \mathcal{N} \mathcal{E}^{\left(\tilde{V}_{i}, V_{-i}\right)}\left(\tilde{V}_{i}, V_{-i}\right)$,

- and player $\tilde{V}_{i}$ misreporting, i.e. $\tilde{s}^{* *} \in \mathcal{N} \mathcal{E}^{\left(\tilde{V}_{i}, V_{-i}\right)}\left(V_{i}, V_{-i}\right)$.

How does Nash-MON work? The key idea is that the marginal gain from reporting truthfully must always be greater than the marginal gain from misreporting, and this must be true for all (relevant) Nash equilibria. Why is Nash-MON a monotonicity condition? The reason is made clearer below (that 
is, after introducing additional assumptions). But for now, suppose that $V_{i}$ is a 'higher' type that $\tilde{V}_{i}$. Then Nash-MON can be viewed as requiring that the marginal gain of matching $V_{i}$ with a 'better' outcome-that is $f\left(V_{i}, V_{-i}\right)$-is greater than matching $\tilde{V}_{i}$ with a 'worse' outcome-that is $f\left(\tilde{V}_{i}, V_{-i}\right)$. We refer the interested reader to Examples 3 and 4, where the role of matching higher types with better outcomes is clearly demonstrated.

It is important to note that Nash-MON only checks those Nash equilibria that are relevant for players' true types. This is closely related to the assumption that players' true types are revealed after the mechanism designer makes a social decision. What would happen if players' types are not revealed (which would be akin to firms entering the oil market without knowing other firms' types)? In general, an entirely different condition than Nash-MON would be required. One would not only need to check conditions for the true Nash equilibria but also all possible Nash equilibria that could emerge for any combination of players. This would be a much stronger condition than Nash-MON, and it is unlikely that the positive results that are shown below would be possible.

It is straightforward to show that Nash-MON is a necessary condition for implementation in any environment with ex post hidden actions.

Lemma 1 (Necessity of Nash-MON) In an environment with ex post hidden actions, a social choice function $f$ is truthful only if $f$ satisfies Nash-MON.

Proof of Lemma 1. Suppose that $f: \mathcal{D} \rightarrow Q$ is truthful, and let $p: \mathcal{D} \rightarrow \mathbb{R}^{n}$ implement $f$. Consider agent $i \in \mathcal{N}$. Fix $V_{-i} \in \mathcal{D}$. Consider types $V_{i}, \tilde{V}_{i} \in \mathcal{D}_{i}$ that yield different outcomes $\left(f\left(V_{i}, V_{-i}\right) \neq f\left(\tilde{V}_{i}, V_{-i}\right)\right)$. If type $V_{i}$ participates in the social choice mechanism $(f, p)$, then Nash DSIC implies that

$$
V_{i}\left(f\left(V_{i}, V_{-i}\right), s^{*}\right)-p_{i}\left(V_{i}, V_{-i}\right) \geq V_{i}\left(f\left(\tilde{V}_{i}, V_{-i}\right), s^{* *}\right)-p_{i}\left(\tilde{V}_{i}, V_{-i}\right)
$$

for all $s^{*} \in \mathcal{N} \mathcal{E}^{\left(V_{i}, V_{-i}\right)}\left(V_{i}, V_{-i}\right)$ and $s^{* *} \in \mathcal{N} \mathcal{E}^{\left(V_{i}, V_{-i}\right)}\left(\tilde{V}_{i}, V_{-i}\right)$ (supposing that such equilibria exist). If type $\tilde{V}_{i}$ participates in $(f, p)$, then Nash DSIC implies that

$$
\tilde{V}_{i}\left(f\left(\tilde{V}_{i}, V_{-i}\right), \tilde{s}^{*}\right)-p_{i}\left(\tilde{V}_{i}, V_{-i}\right) \geq \tilde{V}_{i}\left(f\left(V_{i}, V_{-i}\right), \tilde{s}^{* *}\right)-p_{i}\left(V_{i}, V_{-i}\right)
$$

for all $\tilde{s}^{*} \in \mathcal{N} \mathcal{E}^{\left(\tilde{V}_{i}, V_{-i}\right)}\left(\tilde{V}_{i}, V_{-i}\right)$ and $\tilde{s}^{* *} \in \mathcal{N} \mathcal{E}^{\left(\tilde{V}_{i}, V_{-i}\right)}\left(V_{i}, V_{-i}\right)$ (again supposing that such equilibria exist). Combining (4) and 5 yields

$$
\begin{aligned}
V_{i}\left(f\left(V_{i}, V_{-i}\right), s^{*}\right)- & V_{i}\left(f\left(\tilde{V}_{i}, V_{-i}\right), s^{* *}\right) \\
& \geq p_{i}\left(V_{i}, V_{-i}\right)-p_{i}\left(\tilde{V}_{i}, V_{-i}\right) \\
& \geq \tilde{V}_{i}\left(f\left(V_{i}, V_{-i}\right), \tilde{s}^{* *}\right)-\tilde{V}_{i}\left(f\left(\tilde{V}_{i}, V_{-i}\right), \tilde{s}^{*}\right) .
\end{aligned}
$$


Finally, (6) $\geq$ (8) implies that Nash-MON is necessary.

\subsubsection{Nash-MON Alone Is not Sufficient}

Given the many impossibility theorems in implementation theory, it is perhaps unsurprising to find that Nash-MON alone is not sufficient for truthfulness. We show in the following example that, even in a single-agent environment, ex post hidden actions make it impossible to find a payment function that implements a social choice function. ${ }^{5}$

Example 2 (By itself, Nash-MON is not sufficient for truthfulness). Suppose there are three outcomes, $Q=\{A, B, C\}$, and one agent, $\mathcal{N}=\{1\}$. Let the domain of types be $\mathcal{D}_{1}=\left\{V^{A}, V^{B}, V^{C}\right\}$. Let the set of possible ex post hidden actions be $\mathcal{S}_{1}=\left\{s_{1}, s_{2}\right\}$. Consider a social choice function such that $f\left(V^{A}\right)=A, f\left(V^{B}\right)=B$ and $f\left(V^{C}\right)=C$. The agent's preferences over outcomes, as a function of private information, is given as

$$
V^{A}=\begin{gathered}
A \\
B \\
C
\end{gathered}\left[\begin{array}{cc}
s_{1} & s_{2} \\
55 & -5 \\
50 & 40 \\
60 & 70
\end{array}\right] \quad V^{B}=\begin{gathered}
A \\
B \\
C
\end{gathered}\left[\begin{array}{cc}
-10 & 0 \\
25 & 60 \\
70 & 85
\end{array}\right] \quad V^{C}=\begin{gathered}
A \\
B \\
C
\end{gathered}\left[\begin{array}{cc}
0 & -5 \\
40 & 35 \\
75 & 20
\end{array}\right] .
$$

As such, if $V^{A}$ reports truthfully then his/her optimal action is $s_{1}$ and $V^{A}\left(A, s_{1}\right)=0$. If $V^{B}$ reports truthfully then his/her optimal action is $s_{2}$ and $V^{B}\left(B, s_{2}\right)=60$. If $V^{C}$ reports truthfully then his/her optimal action is $s_{1}$ and $V^{C}\left(C, s_{1}\right)=75$.

In a single-agent environment, Nash-MON checks over the agent's optimal action at each outcome, e.g. $s_{1}=\arg \max _{s \in\left\{s_{1}, s_{2}\right\}} V^{A}(A, s)$ and $s_{2}=$ $\arg \max _{s \in\left\{s_{1}, s_{2}\right\}} V^{A}(C, s)$. This means that Nash-MON imposes three conditions:

$$
\begin{aligned}
& V^{A}\left(A, s_{1}\right)-V^{A}\left(B, s_{1}\right)=0-55 \geq 0-60=V^{B}\left(A, s_{2}\right)-V^{B}\left(B, s_{2}\right) \\
& V^{A}\left(A, s_{1}\right)-V^{A}\left(C, s_{2}\right)=0-70 \geq 0-75=V^{C}\left(A, s_{1}\right)-V^{C}\left(C, s_{1}\right) \\
& V^{B}\left(B, s_{2}\right)-V^{B}\left(C, s_{2}\right)=60-85 \geq 40-75=V^{C}\left(B, s_{1}\right)-V^{C}\left(C, s_{1}\right) .
\end{aligned}
$$

As such, Nash-MON is satisfied in this setting.

5 The following example builds on Example 1 from Bikhchandani et al. (2006) directly. 
However, there is no payment function that can implement $f$. Suppose that the agent pays $p^{A}$ for report $V^{A}, p^{B}$ for report $V^{B}$, and $p^{C}$ for report $V^{C}$. Without loss of generality let $p^{A}=0$. If it is optimal for type $V^{A}$ to report truthfully vs. report $V^{B}$ then

$$
V^{A}\left(A, s_{1}\right)-p^{A} \geq V^{A}\left(B, s_{1}\right)-p^{B} \Longrightarrow p^{B} \geq 55 .
$$

If it is optimal for type $V^{B}$ to report truthfully vs. report $V^{C}$ then

$$
V^{B}\left(B, s_{2}\right)-p^{B} \geq V^{B}\left(C, s_{2}\right)-p^{C} \Longrightarrow p^{C}-p^{B} \geq 25 .
$$

Taken together, (9) and (10) imply that $p^{C} \geq 80$. But if type $V^{C}$ prefers reporting truthfully vs. reporting $V^{A}$, then

$V^{C}\left(C, s_{1}\right)-p^{C} \geq V^{C}\left(A, s_{1}\right)-p^{A} \Longleftrightarrow 75-p^{C} \geq 0-0 \Longleftrightarrow 75 \geq p^{C}$

which contradicts $p^{C} \geq 80$. Consequently, even though $f$ satisfies Nash-MON, there exists no payment function that can implement $f$.

Because Nash-MON alone is not sufficient, we pursue two strategies that circumvent the kind of scenarios presented above. The first strategy focuses on unit-demand auctions with identical goods. The second strategy uses restrictions on the domain of types in a similar manner to Bikhchandani et al. (2006).

\subsection{Unit-Demand Auctions with Identical Goods}

Our first result focuses on unit-demand auctions with identical goods, which includes the oil drilling license auction in Example 1 (we show this in Remark 3 after we present the definition of a unit-demand auction).

In a unit-demand auction with identical goods, each agent only cares about acquiring an item and does not necessarily care about which item is acquired. ${ }^{6}$ This means that the space of mechanism outcomes can be represented as $Q \subseteq\{0,1\}^{n}$, where 1 represents an agent receiving an item and 0 represents an agent not receiving an item. Consequently, if other agents report $V_{-i}$, then $i$ 's report can only render two possible mechanism outcomes: report a 'high type' and win an item or report a 'low type' and not win an item.

6 Note that this does not mean that agents are symmetric, or that agents equally value winning an auction. In contrast, it only means that all goods being auctioned can be treated as equivalent. 
To formalize these ideas in a definition, we endow the set of outcomes $Q \subseteq\{0,1\}^{n}$ with an order $\geq_{i}$ that represents whether or not agent $i$ wins an item. That is, for any $q^{\prime}, q^{\prime \prime} \in Q$, we let $q^{\prime} \geq_{i} q^{\prime \prime} \Longleftrightarrow q_{i}^{\prime}>q_{i}^{\prime \prime} \Longleftrightarrow q_{i}^{\prime}=$ $1>0=q_{i}^{\prime \prime}$.

Definition 6 (Unit-Demand Auction with Identical Goods). An environment with ex post hidden actions is a unit-demand auction with identical goods if:

(i) The mechanism outcome space is $Q \subseteq\{0,1\}^{n}$,

(ii) Each agent $i$ can either win or not win an item, i.e. $\mid\left\{f\left(V_{i}, V_{-i}\right): V_{i} \in\right.$ $\left.\mathcal{D}_{i}\right\} \mid=2$ for all $V_{-i} \in \mathcal{D}_{-i}$,

(iii) Each agent prefers winning an item to not winning an item, that is, $\bar{q} \geq_{i} \underline{q}$ implies that $V_{i}(\bar{q}, \bar{s}) \geq V_{i}(\underline{q}, \underline{s})$ for every $\bar{s} \in \mathcal{N} \mathcal{E}^{V}(\bar{q}), \underline{s} \in \mathcal{N} \mathcal{E}^{V}(\underline{q})$ and $V \in \mathcal{D}$.

Remark 3. Under certain restrictions, it is straightforward to show that the oil drilling license auction in Example 1 is a unit-demand auction with identical goods. (i) By definition, the distribution of licenses can be represented as $Q \subseteq\{0,1\}^{n}$. (ii) By setting $\mathcal{D}_{i}=\mathcal{D}_{j}$ for all $i, j \in \mathcal{N}$, it means that a player $i$ may not win a good because he/she may be outbid by player $j$-different type spaces could otherwise make $j$ win de facto. (iii) Finally, by choosing an appropriate pricing function, it can be guaranteed that the Nash equilibrium profits are always greater than zero for winners. This in turn satisfies condition (iii) of Definition 6. It should also be noted that, by choosing the inverse demand function appropriately, it can be guaranteed that there exists a Nash equilibrium at every $\left(V_{i}, V_{-i}\right)$ combination (e.g. see Examples 3 and 4 below).

Our first main result shows that Nash-MON is necessary and sufficient for implementing a social choice function in a unit-demand auction with identical goods (see Appendix A for the proof).

Theorem 1 In a unit-demand auction environment with identical goods and ex post hidden actions, a social choice function is truthful if and only if it satisfies Nash-MON.

There are perhaps two surprising features of Theorem 1 that are worth noting. First, besides (iii) in Definition 6, no other assumptions are made regarding the space of exogenous private information, $\mathcal{D}$, and ex post hidden 
actions, $\mathcal{S}$. This generality is in contrast to most implementation results that impose specific assumptions on the type space (as in Roberts, 1979, and Bikhchandani et al., 2006). Second, the theorem is a 'positive' result for a problem that allows types to be (strategically) interdependent, which contrasts with many negative results in implementation theory, especially when types are interdependent (see, e.g., Jehiel et al., 2006).

Below, we apply Theorem 1 to the oil drilling license auction from above. In Example 3, we show that there exists a truthful mechanism that can allocate licenses to the most production-efficient firms. In Example 4, we show that the welfare maximizing social choice function cannot be implemented, even by the Vickery-Clarke-Groves mechanism.

\section{Example 3 (continued from Example 1: A social choice function that} satisfies Nash-MON). This example builds on the oil drilling license auction from Example 1. To make it more concrete, we assume that the inverse demand function is linear, $P(X)=a-b X$. Then for appropriately selected $(a, b)$, there exists a unique Nash equilibrium.

In this example, we study the efficiency social choice function, $f^{E F F}$ : $\mathcal{D} \rightarrow Q$, which gives licenses to the most production-efficient firms. This means that, if firms report $\left(V_{1}, V_{2}, V_{3}\right)=(4,5,6)$ then $f^{E F F}(4,5,6)=(1,1,0)$ (firm 3 does not receive a license because it reported the highest marginal cost of production). Because this example is a unit-demand auction with identical goods (see Remark 3), it follows from Theorem 1 that $f^{E F F}$ can be implemented if and only if it satisfies Nash-MON.

To see whether $f^{E F F}$ satisfies Nash-MON, fix $V_{-i} \in \mathcal{D}_{-i}$. Consider $V_{i}, \tilde{V}_{i} \in$ $\mathcal{D}_{i}$ such that $f^{E F F}\left(V_{i}, V_{-i}\right)$ gives $i$ an oil drilling license and $f^{E F F}\left(\tilde{V}_{i}, V_{-i}\right)$ does not give $i$ an oil drilling license (which means that $V_{i}<\tilde{V}_{i}$ ). Then Nash-MON is satisfied if and only if

$$
\begin{aligned}
V_{i}\left(f^{E F F}\left(V_{i}, V_{-i}\right),\right. & \left.s^{*}\right)-V_{i}\left(f^{E F F}\left(\tilde{V}_{i}, V_{-i}\right), s^{* *}\right) \\
& \geq \tilde{V}_{i}\left(f^{E F F}\left(V_{i}, V_{-i}\right), \tilde{s}^{* *}\right)-\tilde{V}_{i}\left(f^{E F F}\left(\tilde{V}_{i}, V_{-i}\right), \tilde{s}^{*}\right)
\end{aligned}
$$

where $\left(s^{*}, s^{* *}, \tilde{s}^{* *}, \tilde{s}^{*}\right)$ are the unique Nash equilibria in the oil drilling market in the respective setting (see Definition 5 for the precise definition). Because firm $i$ 's payoff is zero if it does not win a license, (11) reduces to

$$
V_{i}\left(f^{E F F}\left(V_{i}, V_{-i}\right), s^{*}\right) \geq \tilde{V}_{i}\left(f^{E F F}\left(V_{i}, V_{-i}\right), \tilde{s}^{* *}\right)
$$


where $s^{*} \in \mathcal{N} \mathcal{E}^{\left(V_{i}, V_{-i}\right)}\left(V_{i}, V_{-i}\right)$ and $\tilde{s}^{*} \in \mathcal{N} \mathcal{E}^{\left(\tilde{V}_{i}, V_{-i}\right)}\left(V_{i}, V_{-i}\right)$. If $V_{j}$ also wins a license under $f^{E F F}\left(V_{i}, V_{-i}\right)$, then we can plug in $i$ 's Nash equilibrium profits to re-write (12) as

$$
\begin{aligned}
V_{i}\left(f^{E F F}\left(V_{i}, V_{-i}\right), s^{*}\right) & =\frac{\left(a-2 V_{i}+V_{j}\right)^{2}}{9 b} \\
& \left.\geq \frac{\left(a-2 \tilde{V}_{i}+V_{j}\right)^{2}}{9 b}=\tilde{V}_{i}\left(f^{E F F}\left(V_{i}, V_{-i}\right), \tilde{s}^{* *}\right)\right)
\end{aligned}
$$

(this is the standard Nash equilibrium result from a Cournot model competition with a linear inverse demand). Because $V_{i}<\tilde{V}_{i}$ it follows that (13) holds, which means that Nash-MON also holds. It thus follows from Theorem 1 that $f^{E F F}$ can be implemented. We refer the interested reader to the proof of Theorem 1 (Appendix A) where we propose a payment function that implements $f^{E F F}$.

\section{Example 4 (continued from Example 1: Maximizing welfare is not imple-} mentable). This example builds on the previous one. But here, we present a social choice function that cannot be implemented.

Consider the same Cournot entry environment as above, except now, we study whether the welfare-maximizing social choice function $f^{W M}: \mathcal{D} \rightarrow Q$ can be implemented: that is, $f^{W M}$ gives licenses to the two firms that, at the unique Nash equilibrium, maximize total industry profits. Because this setting is a unit-demand auction with identical goods, it follows from Theorem 1 that $f^{W M}$ can be implemented if and only if $f^{W M}$ satisfies Nash-MON.

To see if $f^{W M}$ satisfies Nash-MON, fix $V_{-i} \in \mathcal{D}_{-i}$. Consider $V_{i}, \tilde{V}_{i} \in \mathcal{D}_{i}$ such that $f^{W M}\left(V_{i}, V_{-i}\right)$ gives $i$ an oil drilling license and $f^{W M}\left(\tilde{V}_{i}, V_{-i}\right)$ does not give $i$ an oil drilling license. Following the same steps as in Example 3, Nash-MON holds if and only if

$$
\begin{gathered}
\overbrace{V_{i}\left(f^{W M}\left(V_{i}, V_{-i}\right), s^{*}\right)}^{\text {Profit when } V_{i} \text { reports truthfully and gets a license }} \\
\geq \underbrace{\tilde{V}_{i}\left(f^{W M}\left(V_{i}, V_{-i}\right), \tilde{s}^{* *}\right)}_{\text {Profit when } \tilde{V}_{i} \text { misreports to get a license }}
\end{gathered}
$$

where $\left(s^{*}, \tilde{s}^{* *},\right)$ are the unique Nash equilibria in the oil drilling market with agents $\left(V_{i}, V_{j}\right)$ and $\left(\tilde{V}_{i}, V_{j}\right)$, respectively. As such, Nash-MON boils down 
to requiring that, at the unique Nash equilibrium, reporting truthfully always yields a higher payoff than misreporting in order to get a license.

We claim that (14) does not hold in general. To see this, suppose that $(a, b)=(10,1)$ and $\left(V_{1}, V_{2}, V_{3}\right)=(1,2,4)$. Let $\Pi_{i}^{i j}$ represent the unique Nash equilibrium payoff of agent $i$ if $i$ and $j$ enter the Cournot competition. Suppose that all agents report truthfully. Because

$$
\Pi_{1}^{12}+\Pi_{2}^{12}=16.6<\Pi_{1}^{13}+\Pi_{3}^{13}=17.0>\Pi_{2}^{23}+\Pi_{3}^{23}=13.0
$$

it follows that $f^{W M}\left(V_{1}, V_{2}, V_{3}\right)=(1,0,1)$ (that is, firms 1 and 3 win licenses).

Is there any $\tilde{V}_{3} \in \mathcal{D}_{3} \subseteq \mathbb{R}_{+}$such that (14) does not hold? Consider $\tilde{V}_{3}=3$. Since the unique Nash equilibrium payoffs with types $\left(V_{1}, V_{2}, \tilde{V}_{3}\right)$ are

$$
\Pi_{1}^{12}+\Pi_{2}^{12}=16.6>\Pi_{1}^{1 \tilde{3}}+\Pi_{\tilde{3}}^{1 \tilde{3}}=16.2>\Pi_{2}^{2 \tilde{3}}+\Pi_{\tilde{3}}^{2 \tilde{3}}=13.0
$$

it follows that $f^{W M}\left(V_{1}, V_{2}, \tilde{V}_{3}\right)=(1,1,0)$. However, it is easy to show that (14) is not satisfied with $V_{3}=4$ and $\tilde{V}_{3}=3$ :

$$
V_{3}\left(f^{W M}\left(V_{1}, V_{2}, V_{3}\right), s^{*}\right)=1.0 \nsupseteq 2.8=\tilde{V}_{3}\left(f\left(V_{1}, V_{2}, V_{3}\right), \tilde{s}^{* *}\right)
$$

(1.0 and 2.8 are calculated using (13)). Consequently, follows from Theorem 1 that there exists no payment function $p: \mathcal{D} \rightarrow \mathbb{R}^{3}$ that can implement the welfare-maximizing social choice function, $f^{W M}$.

Remark 4. Because there exists no payment function $p: \mathcal{D} \rightarrow \mathbb{R}^{3}$ that can implement the welfare-maximizing social choice function, it immediately follows that the Vickery-Clarke-Groves Mechanism cannot implement the efficient allocation rule shown above.

\subsection{Generalized Implementation with ex post Hidden Actions}

Here, we explore one way that Theorem 1 can be generalized beyond unitdemand auctions with identical goods. Our strategy for doing so is extending the concept of a "rich" type space introduced by Bikhchandani et al. (2006).

\subsubsection{Restricting the Type Domain, $\mathcal{D}$}

For each agent, we endow the set of mechanism outcomes $Q$ with an order, $\geq_{i}$. We assume that $\left(Q, \geq_{i}\right)$ is a quasi-ordered space, which means that it is: 
(i) reflexive, i.e. $q \geq_{i} q$ for every $q \in Q$, and (ii) transitive, i.e. $q \geq_{i} q^{\prime}$ and $q^{\prime} \geq_{i} q^{\prime \prime} \Longrightarrow q \geq_{i} q^{\prime \prime}$ for every $q, q^{\prime}, q^{\prime \prime} \in Q$. In practice, $\geq_{i}$ represents agent $i$ 's ranking of mechanism outcomes (this is made clear below).

Our notion of a rich environment builds on the idea that all agents prefer 'better' outcomes. To make this more concrete, we introduce an order by which players rank the outcome space.

Definition 7 (Consistency). In an environment with ex post hidden actions, we say that a type $V_{i} \in \mathscr{D}_{i}$ is consistent with $\left(Q, \geq_{i}\right)$ if, for all $V_{-i} \in \mathcal{D}_{-i}$ :

$$
\begin{aligned}
& q_{k} \geq_{i} q_{l} \Longrightarrow V_{i}\left(q_{k}, s^{k}\right) \geq V_{i}\left(q_{l}, s^{l}\right) \text { for all Nash equilibria } \\
& s^{k} \in \mathcal{N} \mathcal{E}^{\left(V_{i}, V_{-i}\right)}\left(q_{k}\right) \text { and } s^{l} \in \mathcal{N} \mathcal{E}^{\left(V_{i}, V_{-i}\right)}\left(q_{l}\right) .
\end{aligned}
$$

In other words, a consistent type will always prefer 'better' mechanism outcomes $\left(q_{k} \geq_{i} q_{l}\right)$ with respect to all relevant Nash equilibria. One can also think about a consistent type as having preferences that follow his/her order over types, $\geq_{i}$.

A rich type space is simply the collection of all consistent types.

Definition 8 (Rich Type Space, $\mathcal{D}_{i}$ ). In an environment with ex post hidden actions, $\mathcal{D}_{i}$ is rich type space if there is a quasi-order $\geq_{i}$ on $Q$ such that every $V_{i} \in \mathscr{D}_{i}$ that is consistent with $\left(Q, \geq_{i}\right)$ is in $\mathcal{D}_{i}$.

A rich environment extends the definition of a rich type space to each agent.

Definition 9 (A Rich Environment). An environment with ex post hidden actions is rich if there are quasi-orders $\left(\geq_{j}\right)_{j \in \mathcal{N}}$ on $Q$ such that each $\mathcal{D}_{j}$ is a rich type space.

Remark 5. It is worth noting the differences between a rich environment and a unit-demand auction (Definition 6). Because of Definition 6(iii), a unit-demand requires that every type $V_{i} \in \mathcal{D}_{i}$ is consistent (this follows from defining a quasi-order that orders an outcome based on whether $i$ wins or does not win an item). However, a unit-demand auction environment does not require the type space to be rich.

While a unit-demand auction environment permits a more general type space, the trade-off comes in the definition of the mechanism outcome space, $Q$. Unit-demand auctions require that $Q \subseteq\{0,1\}^{n}$. Rich environments, however, impose no constraints on $Q$. 
It turns out that 'richness' provides the key to proving the main result of this paper. In the following theorem, we show that Nash-MON is necessary and sufficient for implementation when restricting attention to rich environments (see Appendix B for the proof).

Theorem 2 In a rich environment with ex post hidden actions, a social choice function is truthful if and only if it satisfies Nash-MON.

There are two points worth raising that help unpack Theorem 2. First, Theorem 2 identifies the necessary and sufficient features a social choice function must have if we hope to find a mechanism that implements it. These features — summarized by Nash-MON_boil down to requiring that matching 'higher types' with 'better outcomes' exhibits increasing returns. Without this feature, it is generally impossible to implement a social choice function in a way that is robust with respect to ex post hidden actions.

Second, it is worth asking what kind of payment function can implement social choice functions that satisfy Nash-MON. We propose such a function in the proof of Theorem 2 (see Appendix B). Broadly speaking, the payment function builds on the 'increasing returns' property from Nash-MON and links it to price reports in a way that renders truth-telling as optimal. Also, similar to Bikhchandani et al. (2006), each agent $i$ 's payment function only depends on $V_{-i}$ and the mechanism outcome $f\left(V_{i}, V_{-i}\right)$. This allows us to ensure that truth-telling is not only optimal but is also a dominant-strategy.

\section{CONCLUDING REMARKS}

The key message of this paper is that strategic actions are important for designing institutions that implement societal goals. With a few exceptions, previous mechanism design models do not incorporate strategic actions that influence personal and/or others' preferences. The paper argues that strategic actions matter, and that current mechanisms - such as the Vickery-ClarkeGroves Mechanism - are not guaranteed to be truthful when strategic actions are present.

This paper makes two contributions. The first is introducing ex post hidden actions, which is one way of embedding mechanisms in a large space of real-world environments that the mechanism cannot control. The example used throughout the paper is an auction that is distributing oil drilling licenses, and the 'ex post hidden actions' represent the strategic market decisions that firms 
take after winning oil drilling licenses. The second is characterizing social choice functions that can be implemented in a way that is robust with respect to ex post hidden actions. We complement this characterization by proposing a mechanism that does so. By means of examples, we show that the tools in this paper can be used in applied settings.

We present two characterizations in the main text. The first characterization can be viewed as an 'off-the-shelf' result that can be easily amended to applied economic settings. It applies directly to unit-demand auctions with identical goods and admits almost any combination of agent types and strategic actions. The second can be viewed as one way to generalize the first. This result falls somewhere between adding ex post hidden actions to the model in Bikhchandani et al. (2006) and offering a strategic counterpart to the work by Bergemann \& Morris (2005) on robust mechanism design.

Looking forward, there are several avenues for future work. One possibility is exploring other concepts of truthfulness. This paper focused on designing mechanisms that are robust with respect to Nash equilibrium, but this requires that agents have sufficient information in order to correctly deduce a Nash equilibrium. Different assumptions with weaker information criteria are possible, such as a maximin approach (Wolitzky, 2016). Another possibility is studying the tension between implementation with ex post hidden actions vs. individual rationality (Ma et al., 1988; Jackson \& Palfrey, 2001), which was not touched on in this paper. Finally, there is the possibility of developing more applied-oriented results. This paper showed that, by focusing on unit-demand auctions with identical goods, a sharp and useful characterization was possible. Future work could explore other applied-oriented environments to uncover more economic insights into implementation with ex post hidden actions.

\section{A. PROOF OF THEOREM 1}

The necessity of Nash-MON follows from Lemma 1. Therefore, all that remains is to show that Nash-MON is also sufficient when we restrict attention to unit-demand auction environments with identical goods and ex post hidden actions.

The proof of sufficiency proceeds as follows. First, we define a pricing function. Second, we identify a useful inequality relationship of the pricing function (Lemma A.2). Third, we show that the pricing function is truthful at 'end points' (Lemma A.3). Finally, we build on these two results to show that 
the pricing function implements $f$ (Lemma A.1).

Before we define the pricing function, some definitions are in order.

In what follows, $V_{-i} \in \mathcal{D}_{-i}$ is fixed.

\section{Inverse social choice function}

Let $\mathcal{Y}_{i}\left(\cdot \mid V_{-i}\right): Q \rightrightarrows \mathcal{D}_{i}$ be the inverse social choice function,

$$
\mathcal{Y}_{i}\left(q_{k} \mid V_{-i}\right) \equiv \mathcal{Y}_{i}\left(q_{k}\right)=\left\{V_{i} \in \mathcal{D}_{i}: f\left(V_{i}, V_{-i}\right)=q_{k}\right\}
$$

In other words, $\mathcal{Y}_{i}\left(q_{k} \mid V_{-i}\right) \subseteq \mathcal{D}_{i}$ is the set of all types from $\mathcal{D}_{i}$ such that $f\left(V_{i}, V_{-i}\right)=q_{k}$.

\section{Differencing function}

Consider the following differencing function between any two outcomes $q_{k}, q_{l} \in Q$ that depends on $V_{-i}$ :

$$
\delta_{q_{k} q_{l}}\left(V_{-i}\right)=\left\{\begin{aligned}
\inf \left\{V_{i}\left(q_{k}, s^{k}\right)-V_{i}\left(q_{l}, s^{l}\right): V_{i} \in \mathcal{Y}_{i}\left(q_{k} \mid V_{-i}\right),\right. & \\
\left.s^{k} \in \mathcal{N} \mathcal{E}^{\left(V_{i}, V_{-i}\right)}\left(q_{k}\right), s^{l} \in \mathcal{N} \mathcal{E}^{\left(V_{i}, V_{-i}\right)}\left(q_{l}\right)\right\} & \text { if } q_{k} \neq q_{l} \\
0 & \text { otherwise. }
\end{aligned}\right.
$$

We often let $\delta_{q_{k} q_{l}}\left(V_{-i}\right) \equiv \delta_{k l}\left(V_{-i}\right)$ unless otherwise ambiguous.

\section{Identifying the 'best' and 'worst' outcome}

By assumption, $\left|\left\{f\left(V_{i}, V_{-i}\right): V_{i} \in \mathcal{D}_{i}\right\}\right|=2$ (see (ii) in Definition 6). Let

$$
\left\{\bar{q}\left(V_{-i}\right), \underline{q}\left(V_{-i}\right)\right\}=\left\{f\left(V_{i}, V_{-i}\right): V_{i} \in \mathcal{D}_{i}\right\}
$$

such that, for any $V_{-i} \in \mathcal{D}_{-i}$,

$$
V_{i}\left(\bar{q}\left(V_{-i}\right), \bar{s}\right) \geq V_{i}\left(\underline{q}\left(V_{-i}\right), \underline{s}\right)
$$

for all $\bar{s} \in \mathcal{N} \mathcal{E}^{\left(V_{i}, V_{-i}\right)}\left(\bar{q}\left(V_{-i}\right)\right)$ and $\underline{s} \in \mathcal{N} \mathcal{E}^{\left(V_{i}, V_{-i}\right)}\left(\underline{q}\left(V_{-i}\right)\right)(\bar{q}$ and $\underline{q}$ have this property by (iv) of Definition 6).

In what follows below, we often let $\bar{q}\left(V_{-i}\right) \equiv \bar{q}$ and $\underline{q}\left(V_{-i}\right) \equiv \underline{q}$ unless otherwise ambiguous. 


\section{Pricing function}

Consider the following payment function (where $\left.f\left(V_{i}, V_{-i}\right)=q\right):^{7}$

$$
p_{i}\left(V_{i}, V_{-i}\right) \equiv p_{i}(q)= \begin{cases}-\delta_{\bar{q}\left(V_{-i}\right) f\left(V_{i}, V_{-i}\right)} & \text { if } f\left(V_{i}, V_{-i}\right)=\underline{q}\left(V_{-i}\right) \\ 0 & \text { otherwise. }\end{cases}
$$

What is the intuition underlying $p_{i}\left(V_{i}, V_{-i}\right)$ ? If $V_{i}$ does not win an item (i.e. $\left.f\left(V_{i}, V_{-i}\right)=q\left(V_{-i}\right)\right)$ then $V_{i}$ is compensated according to $-\delta(\geq 0)$. If $V_{i}$ wins an item then $\bar{V}_{i}$ is not compensated $\left(p_{i}=0\right)$.

In the following lemma, we establish that the payment function given by (16) implements $f$.

Lemma A.1 In a unit-demand auction environment with identical goods and ex post hidden actions, $\left(p_{1}, p_{2}, \ldots, p_{n}\right): \mathcal{D} \rightarrow \mathbb{R}^{n}$ as defined in (16) implements $f$ if $f$ satisfies Nash-MON.

It follows directly from Lemma A. 1 that $f$ is truthful. Before we prove this lemma, we require two supporting lemmas.

\section{First preliminary result: Inequality relationship for $\delta$}

In the following lemma, we show that Nash-MON gives $\delta(\cdot)$ an inequality relationship that turns out to be useful for proving Lemma A.1.

Lemma A.2 Let $V_{-i} \in D_{-i}$ and $q \in\left\{f\left(V_{i}, V_{-i}\right): V_{i} \in \mathcal{D}_{i}\right\}$. Then $\delta_{\bar{q} q}\left(V_{-i}\right) \geq$ $-\delta_{q \bar{q}}\left(V_{-i}\right)$ if $f$ satisfies Nash-MON.

Proof of Lemma A.2. If $q=\bar{q}\left(V_{-i}\right)$ then $\delta_{\bar{q}}\left(V_{-i}\right)=0 \geq 0=-\delta_{\bar{q}} \bar{q}\left(V_{-i}\right)$.

Therefore, suppose that $q=\underline{q}\left(V_{-i}\right)$. By Nash-MON,

$$
V_{i}\left(\underline{q}, s^{*}\right)-V_{i}\left(\bar{q}, s^{* *}\right) \geq \tilde{V}_{i}\left(\underline{q}, \tilde{s}^{* *}\right)-\tilde{V}_{i}\left(\bar{q}, \tilde{s}^{*}\right)
$$

7 It is straightforward to show that this payment function is finite for all possible values. Fix $V_{-i} \in \mathcal{D}_{-i}$. First, $p_{i}^{K}=0$. Second, for $k \neq K$, select $V_{i} \in \mathcal{Y}\left(q_{K} \mid V_{-i}\right)$ and $\tilde{V}_{i} \in \mathcal{Y}_{i}\left(q_{k} \mid V_{-i}\right)$, whereby Nash-MON implies that

$$
\infty>V_{i}\left(q_{K}, s^{*}\right)-V_{i}\left(q_{k}, s^{* *}\right) \geq \tilde{V}_{i}\left(q_{K}, \tilde{s}^{* *}\right)-\tilde{V}_{i}\left(q_{k}, \tilde{s}^{*}\right)>-\infty
$$

for all $s^{*} \in \mathcal{N} \mathcal{E}^{\left(V_{i}, V_{-i}\right)}\left(q_{K}\right), s^{* *} \in \mathcal{N} \mathcal{E}^{\left(V_{i}, V_{-i}\right)}\left(q_{k}\right), \tilde{s}^{* *} \in \mathcal{N} \mathcal{E}^{\left(\tilde{V}_{i}, V_{-i}\right)}\left(q_{K}\right)$, and $\tilde{s}^{*} \in$ $\mathcal{N} \mathcal{E}^{\left(\tilde{V}_{i}, V_{-i}\right)}\left(q_{k}\right)$. Therefore, $-\delta_{K k}=p_{i}^{K}$ is finite. 
for all types $V_{i} \in \mathcal{Y}_{i}\left(\underline{q} \mid V_{-i}\right)$ and $\tilde{V}_{i} \in \mathcal{Y}_{i}\left(\bar{q} \mid V_{-i}\right)$, and Nash equilibria $s^{*} \in \mathcal{N} \mathcal{E}^{\left(V_{i}, V_{-i}\right)}(q), s^{* *} \in \mathcal{N} \mathcal{E}^{\left(V_{i}, V_{-i}\right)}(\bar{q}), \tilde{s}^{* *} \in \mathcal{N} \mathcal{E}^{\left(\tilde{V}_{i}, V_{-i}\right)} \in(q)$, and $\tilde{s}^{*} \in$ $\mathcal{N} \mathcal{E}^{\left(\tilde{V}_{i}, V_{-i}\right)}(\bar{q})$. Consequently,

$$
\begin{aligned}
& \delta_{q} \bar{q}\left(V_{-i}\right)= \inf \left\{V_{i}\left(\underline{q}, s^{*}\right)-V_{i}\left(\bar{q}, s^{* *}\right): V_{i} \in \mathcal{Y}_{i}\left(\underline{q} \mid V_{-i}\right),\right. \\
&\left.s^{*} \in \mathcal{N} \mathcal{E}^{\left(V_{i}, V_{-i}\right)}(\underline{q}), s^{* *} \in \mathcal{N} \mathcal{E}^{\left(V_{i}, V_{-i}\right)}(\bar{q})\right\} \\
& \geq \sup \left\{\tilde{V}_{i}\left(\underline{q}, \tilde{s}^{* *}\right)-\tilde{V}_{i}\left(\bar{q}, \tilde{s}^{*}\right): \tilde{V}_{i} \in \mathcal{Y}_{i}\left(\bar{q} \mid V_{-i}\right),\right. \\
&\left.\tilde{s}^{* *} \in \mathcal{N} \mathcal{E}^{\left(\tilde{V}_{i}, V_{-i}\right)}(\underline{q}), \tilde{s}^{*} \in \mathcal{N} \mathcal{E}^{\left(\tilde{V}_{i}, V_{-i}\right)}(\bar{q})\right\} \\
&=-\inf \left\{\tilde{V}_{i}\left(\bar{q}, \tilde{s}^{*}\right)-\tilde{V}_{i}\left(\underline{q}, \tilde{s}^{* *}\right): \tilde{V}_{i} \in \mathcal{Y}_{i}\left(\bar{q} \mid V_{-i}\right),\right. \\
&\left.\tilde{s}^{*} \in \mathcal{N} \mathcal{E}^{\left(\tilde{V}_{i}, V_{-i}\right)}(\bar{q}), \tilde{s}^{* *} \in \mathcal{N} \mathcal{E}^{\left(\tilde{V}_{i}, V_{-i}\right)}(\underline{q})\right\} \\
&=-\delta_{\bar{q} \underline{q}}\left(V_{-i}\right) .
\end{aligned}
$$

\section{Second preliminary result: Implementing the 'end points' of $Q$}

In the following lemma, we show that the pricing function implements $\bar{q}$ and $\underline{q}$ (the proof of Lemma A.1 is a small step after this result).

Lemma A.3 If $f$ satisfies Nash-MON then for any types $\left(V_{i}, V_{-i}\right) \in \mathcal{D}$, and corresponding Nash equilibria $\underline{s} \in \mathcal{N} \mathcal{E}^{\left(V_{i}, V_{-i}\right)}\left(\underline{q}\left(V_{-i}\right)\right)$ and $\bar{s} \in \mathcal{N} \mathcal{E}^{\left(V_{i}, V_{-i}\right)}\left(\bar{q}\left(V_{-i}\right)\right)$ :

(i) if $V_{i}(\underline{q}, \underline{s})-p_{i}(\underline{q})<V_{i}(\bar{q}, \bar{s})-p_{i}(\bar{q})$ then $f\left(V_{i}, V_{-i}\right) \neq \underline{q}\left(V_{-i}\right)$;

(ii) if $V_{i}(\underline{q}, \underline{s})-p_{i}(\underline{q})>V_{i}(\bar{q}, \bar{s})-p_{i}(\bar{q})$ then $f\left(V_{i}, V_{-i}\right) \neq \bar{q}\left(V_{-i}\right)$.

Proof of Lemma A.3. We prove each claim separately.

\section{Proof of claim (i)}

By way of contradiction, suppose that $f\left(V_{i}, V_{-i}\right)=\underline{q}$. Because $p_{i}(\underline{q})=-\delta_{\bar{q} \underline{q}}$ and $p_{i}(\bar{q})=0$,

$$
V_{i}(\underline{q}, \underline{s})-V_{i}(\bar{q}, \bar{s})<p_{i}(\underline{q})-p_{i}(\bar{q})=\underbrace{-\delta_{\bar{q} \underline{q}}\left(V_{-i}\right) \leq \delta_{\underline{q} \bar{q}}\left(V_{-i}\right)}_{\text {Lemma A.2 }}
$$

Journal of Mechanism and Institution Design 5(1), 2020 
where the last inequality follows from Lemma A.2. Consequently,

$$
\begin{aligned}
& V_{i}(\underline{q}, \underline{s})-V_{i}(\bar{q}, \bar{s})<\delta_{\underline{q} \bar{q}}\left(V_{-i}\right) \\
& =\inf \left\{V_{i}^{\prime}\left(\underline{q}, \underline{s}^{\prime}\right)-V_{i}^{\prime}\left(\bar{q}, \bar{s}^{\prime}\right): V_{i}^{\prime} \in \mathcal{Y}_{i}\left(\underline{q} \mid V_{-i}\right)\right. \text {, } \\
& \left.\underline{s}^{\prime} \in \mathcal{N} \mathcal{E}^{\left(V_{i}^{\prime}, V_{-i}\right)}(\underline{q}), \bar{s}^{\prime} \in \mathcal{N} \mathcal{E}^{\left(V_{i}^{\prime}, V_{-i}\right)}(\bar{q})\right\} \\
& \leq V_{i}(\underline{q}, \underline{s})-V_{i}(\bar{q}, \bar{s})
\end{aligned}
$$

where the last inequality follows because $f\left(V_{i}, V_{-i}\right)=q \Longrightarrow V_{i} \in \mathcal{Y}_{i}\left(\underline{q} \mid V_{-i}\right)$. However, $V_{i}(\underline{q}, \underline{s})-V_{i}(\bar{q}, \bar{s})<V_{i}(\underline{q}, \underline{s})-V_{i}(\bar{q}, \bar{s})$ is an impossibility. Therefore, $f\left(V_{i}, V_{-i}\right) \neq \underline{q}$.

\section{Proof of claim (ii)}

The proof proceeds in a similar manner. By way of contradiction, suppose that $f\left(V_{i}, V_{-i}\right)=\bar{q}$. By assumption,

$$
V_{i}(\bar{q}, \bar{s})-V_{i}(\underline{q}, \underline{s})<p_{i}(\bar{q})-p_{i}(\underline{q})=\delta_{\bar{q} \underline{q}}\left(V_{-i}\right) .
$$

Consequently,

$$
\begin{aligned}
V_{i}(\bar{q}, \bar{s})-V_{i}(\underline{q}, \underline{s}) & <\delta_{\bar{q} \underline{q}}\left(V_{-i}\right) \\
& =\inf \left\{V_{i}^{\prime}\left(\bar{q}, \bar{s}^{\prime}\right)-V_{i}^{\prime}\left(\underline{q}, \underline{s}^{\prime}\right): V_{i}^{\prime} \in \mathcal{Y}_{i}\left(\bar{q} \mid V_{-i}\right),\right. \\
& \left.\bar{s}^{\prime} \in \mathcal{N} \mathcal{E}^{\left(V_{i}^{\prime}, V_{-i}\right)}(\bar{q}), \underline{s}^{\prime} \in \mathcal{N}^{\left(V_{i}^{\prime}, V_{-i}\right)}(\underline{q})\right\} \\
& \leq V_{i}(\bar{q}, \bar{s})-V_{i}(\underline{q}, \underline{s})
\end{aligned}
$$

where the last inequality follows because $f\left(V_{i}, V_{-i}\right)=\bar{q} \Longrightarrow V_{i} \in \mathcal{Y}_{i}\left(\bar{q} \mid V_{-i}\right)$. However, $V_{i}(\bar{q}, \bar{s})-V_{i}(\underline{q}, \underline{s})<V_{i}(\bar{q}, \bar{s})-V_{i}(\underline{q}, \underline{s})$ is an impossibility. Therefore, $f\left(V_{i}, V_{-i}\right) \neq \bar{q}$.

\section{Finishing the proof of Theorem 1}

We conclude the proof of Theorem 1 by proving Lemma A.1. 
Proof of Lemma A.1. We show that $p$ implements $f$. By way of contradiction, suppose that there exists $V_{-i} \in \mathcal{D}_{-i}$, outcomes $q^{\prime}, q^{\prime \prime} \in\left\{f\left(V_{i}^{\prime}, V_{-i}\right): V_{i} \in \mathcal{D}_{i}\right\}$ such that $q^{\prime} \neq q^{\prime \prime}$, a type $V_{i} \in \mathcal{Y}_{i}\left(q^{\prime} \mid V_{-i}\right)$ that implements $q^{\prime}$, a type $\tilde{V}_{i} \in \mathcal{Y}_{i}\left(q^{\prime \prime} \mid V_{-i}\right)$ that implements $q^{\prime \prime}$, and Nash equilibria $s^{\prime} \in \mathcal{N} \mathcal{E}^{\left(V_{i}, V_{-i}\right)}\left(q^{\prime}\right)$ and $s^{\prime \prime} \in \mathcal{N} \mathcal{E}^{\left(V_{i}, V_{-i}\right)}\left(q^{\prime \prime}\right)$ such that

$$
\begin{aligned}
V_{i}\left(q^{\prime}, s^{\prime}\right)-p_{i}\left(V_{i}, V_{-i}\right) & <V_{i}\left(q^{\prime \prime}, s^{\prime \prime}\right)-p_{i}\left(\tilde{V}_{i}, V_{-i}\right) \\
\Longleftrightarrow \quad V_{i}\left(q^{\prime}, s^{\prime}\right)-p_{i}\left(q^{\prime}\right) & <V_{i}\left(q^{\prime \prime}, s^{\prime \prime}\right)-p_{i}\left(q^{\prime \prime}\right)
\end{aligned}
$$

In other words, (17) means that $V_{i}$ can strictly gain by reporting $\tilde{V}_{i}\left(\neq V_{i}\right)$ in order to induce outcome $q^{\prime \prime}$ and Nash equilibrium $s^{\prime \prime}$.

We must consider two possibilities:

(i) Suppose that $q^{\prime \prime}=\bar{q}\left(V_{-i}\right)$. Then $q^{\prime}=q\left(V_{-i}\right)$. By Lemma A.3(i), $V_{i}\left(q, s^{\prime}\right)-p_{i}(q)<V_{i}\left(\bar{q}, s^{\prime \prime}\right)-p_{i}(\bar{q})$ implies that $f\left(V_{i}, V_{-i}\right) \neq q\left(V_{-i}\right)=q^{\prime}$. But then $V_{i} \notin \mathcal{Y}_{i}\left(q^{\prime} \mid V_{-i}\right)$, which is a contradiction.

(ii) Suppose that $q^{\prime}=\bar{q}\left(V_{-i}\right)$. Then $q^{\prime \prime}=q\left(V_{-i}\right)$. By Lemma A.3(ii), $V_{i}\left(\bar{q}, s^{\prime}\right)-p_{i}(\bar{q})<V_{i}\left(q, s^{\prime \prime}\right)-p_{i}(q)$ implies that $f\left(V_{i}, V_{-i}\right) \neq \bar{q}\left(V_{-i}\right)=q^{\prime}$. But then $V_{i} \notin \mathcal{Y}_{i}\left(q^{\prime} \mid \bar{V}_{-i}\right)$, which is a contradiction.

Taken together, $(i)$ and $(i i)$ imply that $q^{\prime}, q^{\prime \prime} \neq \bar{q}\left(V_{-i}\right)$. However, having assumed that $f\left(V_{i}, V_{-i}\right) \neq f\left(\tilde{V}_{i}, V_{-i}\right)$, it must be that $q^{\prime}=\bar{q}\left(V_{-i}\right)$ or $q^{\prime \prime}=\bar{q}\left(V_{-i}\right)$. This impossibility means that $p$ implements $f$.

\section{B. PROOF OF THEOREM 2}

The necessity of Nash-MON follows from Lemma 1. Therefore, all that remains is to prove that Nash-MON is also sufficient when each agent's type space is rich.

The proof of sufficiency proceeds in a similar manner to that shown above. First, we propose a pricing function. Second, we identify two useful inequality relationships of the pricing function (Lemma B.2). Third, we show that the pricing function implements the 'end points' of $Q$ (Lemma B.3). Finally, we build on these results to show that the pricing function implements $f$ (Lemma B.1).

Before we define the pricing function, some definitions are in order.

In what follows, $V_{-i} \in \mathcal{D}_{-i}$ is fixed. 


\section{Inverse social choice function}

Let $\mathcal{Y}_{i}\left(\cdot \mid V_{-i}\right): Q \rightrightarrows \mathcal{D}_{i}$ be the inverse social choice function:

$$
\mathcal{Y}_{i}\left(q_{k} \mid V_{-i}\right)=\left\{V_{i} \in \mathcal{D}_{i}: f\left(V_{i}, V_{-i}\right)=q_{k}\right\} .
$$

In other words, $\mathcal{Y}_{i}\left(q_{k} \mid V_{-i}\right)$ are all the types in $\mathcal{D}_{i}$ such that $f\left(V_{i}, V_{-i}\right)=q_{k}$.

\section{Differencing function between outcomes}

Consider the following differencing function between any two outcomes $q_{k}, q_{l} \in Q$ (which is similar to that defined in (15)):

$$
\delta_{q_{k} q_{l}}\left(V_{-i}\right)=\left\{\begin{array}{cc}
\inf \left\{V_{i}\left(q_{k}, s^{k}\right)-V_{i}\left(q_{l}, s^{l}\right): V_{i} \in \mathcal{Y}_{i}\left(q_{k} \mid V_{-i}\right),\right. & \\
\left.s^{k} \in \mathcal{N} \mathcal{E}^{\left(V_{i}, V_{-i}\right)}\left(q_{k}\right), s^{l} \in \mathcal{N} \mathcal{E}^{\left(V_{i}, V_{-i}\right)}\left(q_{l}\right)\right\} & \text { if } q_{k} \neq q_{l} \\
0 & \text { otherwise. }
\end{array}\right.
$$

\section{Payment function}

Let $\left(\geq_{j}\right)_{j \in \mathcal{N}}$ be the quasi-orders on $Q$ such that $\mathcal{D}_{j}$ is a rich type space (such $\left(\geq_{j}\right)_{j \in \mathcal{N}}$ exist by assumption). For every $V_{-i} \in \mathcal{D}_{-i}$, and with relabeling if necessary, let $q_{K} \in Q$ denote an outcome in $Q$ such that:

(i) $q_{K} \in\left\{f\left(V_{i}, V_{-i}\right): V_{i} \in \mathcal{D}_{i}\right\}$ and

(ii) $q \nsucceq_{i} q_{K}$ for each $q \in\left\{f\left(V_{i}, V_{-i}\right): V_{i} \in \mathcal{D}_{i}\right\} \backslash\left\{q_{K}\right\}$.

The interpretation is that $q_{K}$ is the 'best' possible outcome that $V_{i}$ could obtain given that others reported $V_{-i}$.

Consider the following payment function (supposing that $\left.f\left(V_{i}, V_{-i}\right)=q_{k}\right):^{8}$

$$
p_{i}\left(V_{i}, V_{-i}\right) \equiv p_{i}\left(q_{k}, V_{-i}\right) \equiv p_{i}^{k}= \begin{cases}-\delta_{K k}\left(V_{-i}\right) & \text { if } k \neq K \\ 0 & \text { otherwise. }\end{cases}
$$

In the following lemma, we claim that this payment function implements $f$.

Lemma B.1 In a rich environment with ex post hidden actions, $\left(p_{1}, p_{2}, \ldots, p_{n}\right)$ : $\mathcal{D} \rightarrow \mathbb{R}^{n}$ as defined in (18) implements $f$ if $f$ satisfies Nash-MON.

It follows directly from Lemma B.1 that $f$ is truthful. But to prove Lemma B.1, two additional lemmas are needed.

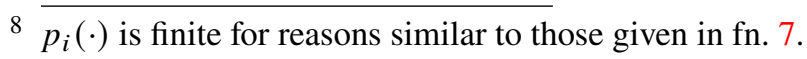




\section{First supporting lemma: Inequality relationships of $\delta$}

It turns out that the following inequality relationships are essential for proving that $\left(p_{1}, \ldots, p_{n}\right): \mathcal{D} \rightarrow \mathbb{R}^{n}$ from (18) implements $f$.

Lemma B.2 For any social choice function $f, V_{-i} \in \mathcal{D}_{-i}$, and $q_{k}, q_{l}, q_{r} \in Q$ :

(i) if $q_{k} \geq_{i} q_{l}$ then $\delta_{r k}\left(V_{-i}\right) \leq \delta_{r l}\left(V_{-i}\right)$;

(ii) if $f$ satisfies Nash-MON then $\delta_{k l}\left(V_{-i}\right) \geq-\delta_{l k}\left(V_{-i}\right)$.

Proof of Lemma B.2. We prove each claim separately.

\section{Proof of claim (i)}

By the rich domain assumption, $V_{i}\left(q_{k}, s^{k}\right) \geq V_{i}\left(q_{l}, s^{l}\right)$ for all types $V_{i} \in \mathcal{D}_{i}$ and Nash equilibria $s^{k} \in \mathcal{N} \mathcal{E}^{\left(V_{i}, V_{-i}\right)}\left(q_{k}\right)$ and $s^{l} \in \mathcal{N} \mathcal{E}^{\left(V_{i}, V_{-i}\right)}\left(q_{l}\right)$. Consequently,

$$
V_{i}\left(q_{r}, s^{r}\right)-V_{i}\left(q_{l}, s^{l}\right) \geq V_{i}\left(q_{r}, s^{r}\right)-V_{i}\left(q_{k}, s^{k}\right)
$$

for all $V_{i} \in \mathcal{Y}_{i}\left(q_{r} \mid V_{-i}\right)$ and $s^{r} \in \mathcal{N} \mathcal{E}^{\left(V_{i}, V_{-i}\right)}\left(q_{r}\right)$. Therefore, $\delta_{r l}\left(V_{-i}\right) \geq$ $\delta_{r k}\left(V_{-i}\right)$.

\section{Proof of claim (ii)}

If $q_{k}$ and $q_{l}$ represent the same outcome then the result is trivial.

Therefore, suppose that $q_{k}$ and $q_{l}$ are different outcomes. By Nash-MON,

$$
V_{i}\left(q_{k}, s^{*}\right)-V_{i}\left(q_{l}, s^{* *}\right) \geq \tilde{V}_{i}\left(q_{k}, \tilde{s}^{* *}\right)-\tilde{V}_{i}\left(q_{l}, \tilde{s}^{*}\right)
$$

for all types $V_{i} \in \mathcal{Y}_{i}\left(q_{k} \mid V_{-i}\right)$ and $\tilde{V}_{i} \in \mathcal{Y}_{i}\left(q_{l} \mid V_{-i}\right)$, and all Nash equilibria $s^{*} \in \mathcal{N} \mathcal{E}^{\left(V_{i}, V_{-i}\right)}\left(q_{k}\right), s^{* *} \in \mathcal{N} \mathcal{E}^{\left(V_{i}, V_{-i}\right)}\left(q_{l}\right), \tilde{s}^{* *} \in \mathcal{N} \mathcal{E}^{\left(\tilde{V}_{i}, V_{-i}\right)}\left(q_{k}\right)$ and $\tilde{s}^{*} \in$ Journal of Mechanism and Institution Design 5(1), 2020 
$\mathcal{N} \mathcal{E}^{\left(\tilde{V}_{i}, V_{-i}\right)}\left(q_{l}\right)$. Consequently,

$$
\begin{aligned}
& \delta_{k l}\left(V_{-i}\right)= \inf \left\{V_{i}\left(q_{k}, s^{*}\right)-V_{i}\left(q_{l}, s^{* *}\right): V_{i} \in \mathcal{Y}_{i}\left(q_{k} \mid V_{-i}\right),\right. \\
&\left.s^{*} \in \mathcal{N} \mathcal{E}^{\left(V_{i}, V_{-i}\right)}\left(q_{k}\right), s^{* *} \in \mathcal{N} \mathcal{E}^{\left(V_{i}, V_{-i}\right)}\left(q_{l}\right)\right\} \\
& \geq \sup \left\{\tilde{V}_{i}\left(q_{k}, \tilde{s}^{* *}\right)-\tilde{V}_{i}\left(q_{l}, \tilde{s}^{*}\right): \tilde{V}_{i} \in \mathcal{Y}_{i}\left(q_{l} \mid V_{-i}\right),\right. \\
&\left.\tilde{s}^{* *} \in \mathcal{N} \mathcal{E}^{\left(\tilde{V}_{i}, V_{-i}\right)}\left(q_{k}\right), \tilde{s}^{*} \in \mathcal{N} \mathcal{E}^{\left(\tilde{V}_{i}, V_{-i}\right)}\left(q_{l}\right)\right\} \\
&=-\inf \left\{\tilde{V}_{i}\left(q_{l}, \tilde{s}^{*}\right)-\tilde{V}_{i}\left(q_{k}, \tilde{s}^{* *}\right): \tilde{V}_{i} \in \mathcal{Y}_{i}\left(q_{l} \mid V_{-i}\right),\right. \\
&\left.\tilde{s}^{*} \in \mathcal{N} \mathcal{E}^{\left(\tilde{V}_{i}, V_{-i}\right)}\left(q_{l}\right), \tilde{s}^{* *} \in \mathcal{N} \mathcal{E}^{\left(\tilde{V}_{i}, V_{-i}\right)}\left(q_{k}\right)\right\}
\end{aligned}
$$

\section{Second supporting lemma: Implementing $q_{K}$}

In the following lemma, we show that the pricing function implements $q_{K}$.

Lemma B.3 Let $f$ be a social choice function that satisfies Nash-MON. Then for any outcome $q_{l} \in Q$ such that $q_{l} \neq q_{K}$, types $\left(V_{i}, V_{-i}\right) \in \mathcal{D}$, and Nash equilibria $s^{l} \in \mathcal{N} \mathcal{E}^{\left(V_{i}, V_{-i}\right)}\left(q_{l}\right)$ and $s^{K} \in \mathcal{N} \mathcal{E}^{\left(V_{i}, V_{-i}\right)}\left(q_{K}\right)$ :

(i) if $V_{i}\left(q_{l}, s^{l}\right)-p_{i}^{l}<V_{i}\left(q_{K}, s^{K}\right)-p_{i}^{K}$ then $f\left(V_{i}, V_{-i}\right) \neq q_{l}$;

(ii) if $V_{i}\left(q_{l}, s^{l}\right)-p_{i}^{l}>V_{i}\left(q_{K}, s^{K}\right)-p_{i}^{K}$ then $f\left(V_{i}, V_{-i}\right) \neq q_{K}$.

Proof of Lemma B.3. We prove each claim separately.

\section{Proof of claim (i)}

By way of contradiction, suppose that $f\left(V_{i}, V_{-i}\right)=q_{l}$. By definition, $p_{i}^{K}=0$ and $p_{i}^{l}=-\delta_{K l}\left(V_{-i}\right)$. Therefore,

$$
V_{i}\left(q_{l}, s^{l}\right)-V_{i}\left(q_{K}, s^{K}\right)<p_{i}^{l}-p_{i}^{K}=\underbrace{-\delta_{K l}\left(V_{-i}\right) \leq \delta_{l K}\left(V_{-i}\right)}_{\text {Lemma B.2(ii) }}
$$


where the last inequality follows from Lemma B.2(ii). Because $f\left(V_{i}, V_{-i}\right)=$ $q_{l} \Longrightarrow V_{i} \in \mathcal{Y}_{i}\left(q_{l} \mid V_{-i}\right)$, it follows that

$$
\begin{aligned}
& V_{i}\left(q_{l}, s^{l}\right)-V_{i}\left(q_{K}, s^{K}\right) \\
& <\delta_{l K}\left(V_{-i}\right) \\
& =\inf \left\{V_{i}^{\prime}\left(q_{l}, \hat{s}^{l}\right)-V_{i}^{\prime}\left(q_{K}, \hat{s}^{K}\right): V_{i}^{\prime} \in \mathcal{Y}_{i}\left(q_{l} \mid V_{-i}\right),\right. \\
& \left.\quad \hat{s}^{l} \in \mathcal{N} \mathcal{E}^{\left(V_{i}^{\prime}, V_{-i}\right)}\left(q_{l}\right), \hat{s}^{K} \in \mathcal{N} \mathcal{E}^{\left(V_{i}^{\prime}, V_{-i}\right)}\left(q_{K}\right)\right\} \\
& \leq V_{i}\left(q_{l}, s^{l}\right)-V_{i}\left(q_{K}, s^{K}\right),
\end{aligned}
$$

where $(19)<(23)=(19)$ is a contradiction. Therefore, $f\left(V_{i}, V_{-i}\right) \neq q_{l}$.

\section{Proof of claim (ii)}

By way of contradiction, suppose that $f\left(V_{i}, V_{-i}\right)=q_{K}$. By assumption,

$$
V_{i}\left(q_{K}, s^{K}\right)-V_{i}\left(q_{l}, s^{l}\right)<p_{i}^{K}-p_{i}^{l}=\delta_{K l}\left(V_{-i}\right) .
$$

Because $f\left(V_{i}, V_{-i}\right)=q_{K} \Longrightarrow V_{i} \in \mathcal{Y}_{i}\left(q_{K} \mid V_{-i}\right)$, it follows that

$$
\begin{aligned}
V_{i}\left(q_{K}, s^{K}\right)-V_{i}\left(q_{l}, s^{l}\right) & \\
< & \delta_{K l}\left(V_{-i}\right) \\
= & \inf \left\{\begin{aligned}
&\{\left(q_{K}, \hat{s}^{K}\right)-V_{i}^{\prime}\left(q_{l}, \hat{s}^{l}\right): V_{i}^{\prime} \in \mathcal{Y}_{i}\left(q_{K} \mid V_{-i}\right), \\
&\left.\hat{s}^{K} \in \mathcal{N} \mathcal{E}^{\left(V_{i}^{\prime}, V_{-i}\right)}\left(q_{K}\right), \hat{s}^{l} \in \mathcal{N} \mathcal{E}^{\left(V_{i}^{\prime}, V_{-i}\right)}\left(q_{l}\right)\right\}
\end{aligned}\right. \\
\leq & V_{i}\left(q_{K}, s^{K}\right)-V_{i}\left(q_{l}, s^{l}\right),
\end{aligned}
$$

where $(24)<(28)=(24)$ is a contradiction. Therefore, $f\left(V_{i}, V_{-i}\right) \neq q_{K}$.

\section{Finishing the proof of Theorem 2}

We conclude the proof of Theorem 2 by proving Lemma B.1.

Proof of Lemma B.1. Fix $V_{-i} \in \mathcal{D}_{-i}$. By way of contradiction, suppose there exist outcomes $q_{j}, q_{k} \in Q\left(q_{j} \neq q_{k}\right)$, a type $V_{i} \in \mathcal{Y}_{i}\left(q_{j} \mid V_{-i}\right)$ that Journal of Mechanism and Institution Design 5(1), 2020 
implements $q_{j}$, a type $\tilde{V}_{i} \in \mathcal{Y}_{i}\left(q_{k} \mid V_{-i}\right)$ that implements $q_{k}$, and Nash equilibria $s^{j} \in \mathcal{N} \mathcal{E}^{\left(V_{i}, V_{-i}\right)}\left(q_{j}\right)$ and $s^{k} \in \mathcal{N} \mathcal{E}^{\left(V_{i}, V_{-i}\right)}\left(q_{k}\right)$ such that

$$
\begin{aligned}
& V_{i}\left(q_{j}, s^{j}\right)-p_{i}\left(V_{i}, V_{-i}\right)<V_{i}\left(q_{k}, s^{k}\right)-p_{i}\left(\tilde{V}_{i}, V_{-i}\right) \\
& \Longleftrightarrow V_{i}\left(q_{j}, s^{j}\right)-p_{i}^{j}<V_{i}\left(q_{k}, s^{k}\right)-p_{i}^{k} .
\end{aligned}
$$

In other words, (29) says that $V_{i}$ can strictly gain by reporting $\tilde{V}_{i}\left(\neq V_{i}\right)$ in order to induce outcome $q_{k}$ and Nash equilibrium $s^{k}$.

It follows that $k \neq K$; otherwise by Lemma B.3(i), $V_{i}\left(q_{j}, s^{j}\right)-p_{i}^{j}<$ $V_{i}\left(q_{K}, s^{K}\right)-p_{i}^{K}$ implies $f\left(V_{i}, V_{-i}\right) \neq q_{j}$, which contradicts the initial assumption. Similarly, it also follows that $j \neq K$; otherwise by Lemma B.3(ii), $V_{i}\left(q_{K}, s^{K}\right)-p_{i}^{K}<V_{i}\left(q_{k}, s^{k}\right)-p_{i}^{k}$ implies $f\left(V_{i}, V_{-i}\right) \neq q_{K}$, which contradicts the initial assumption.

Since $f\left(V_{i}, V_{-i}\right)=q_{j}$, it follows from the contrapositive of Lemma B.3(i) that $V_{i}\left(q_{j}, s^{j}\right)-p_{i}^{j} \geq V_{i}\left(q_{K}, s^{K}\right)-p_{i}^{K}$ for any $s^{K} \in \mathcal{N} \mathcal{E}^{\left(V_{i}, V_{-i}\right)}\left(q_{K}\right)$. Consider $\gamma>\varepsilon>0$ such that

$$
V_{i}\left(q_{j}, s^{j}\right)+\varepsilon-p_{i}^{j}<V_{i}\left(q_{K}, s^{K}\right)+\gamma-p_{i}^{K}<V_{i}\left(q_{k}, s^{k}\right)-p_{i}^{k} .
$$

Define

$$
\widehat{Q}=\left\{q_{j}\right\} \cup\left\{q_{l} \in Q: q_{l} \geq_{i} q_{j} \text { and } \max _{s^{l} \in \mathcal{N} \mathcal{E}^{\left(V_{i}, V_{-i}\right)}\left(q_{l}\right)} V_{i}\left(q_{l}, s^{l}\right)=V_{i}\left(q_{j}, s^{j}\right)\right\} .
$$

Define the type $\widehat{V}_{i}$ in the following way:

$$
\widehat{V}_{i}\left(q_{r}, s\right)= \begin{cases}V_{i}\left(q_{j}, s^{j}\right)+\varepsilon & \text { if } q_{r} \in \widehat{Q} \backslash\left\{q_{K}\right\} \\ \max _{s^{r} \in \mathcal{N} \mathcal{E}^{\left(V_{i}, V_{-i}\right)}\left(q_{r}\right)} V_{i}\left(q_{r}, s^{r}\right)+\gamma & \text { if } q_{r}=q_{K} \\ \max _{s^{r} \in \mathcal{N} \mathcal{E}^{\left(V_{i}, V_{-i}\right)}\left(q_{r}\right)} V_{i}\left(q_{r}, s^{r}\right) & \text { otherwise. }\end{cases}
$$

Note that, for any $q_{l} \in \widehat{Q}$, any $\mathcal{N} \mathcal{E}^{\left(V_{i}, V_{-i}\right)}\left(q_{l}\right)=\mathcal{N} \mathcal{E}^{\left(\widehat{V}_{i}, V_{-i}\right)}\left(q_{l}\right)$.

We make the following claim about $\widehat{V}_{i}$.

Claim $1 \widehat{V}_{i}$ as defined in (31) is consistent with $\left(Q, \geq_{i}\right)$.

Supposing Claim 1 is true (we prove this below), it follows from the rich environment assumption that $\widehat{V}_{i} \in \mathcal{D}_{i}$. Since $q_{l} \geq_{i} q_{j}$ for all $q_{l} \in \widehat{Q}$, it follows from Lemma B.2(i) that $\delta_{K l}\left(V_{-i}\right) \leq \delta_{K j}\left(V_{-i}\right)$ and $p_{i}^{l} \geq p_{i}^{j}$ for all 
$q_{l} \in \widehat{Q}$. Because $\max _{s^{l} \in \mathcal{N} \mathcal{E}^{\left(V_{i}, V_{-i}\right)}\left(q_{l}\right)} V_{i}\left(q_{l}, s^{l}\right)=\widehat{V}_{i}\left(q_{l}, \bar{s}^{l}\right)=\widehat{V}_{i}\left(q_{j}, s^{j}\right)$ for all $q_{l} \in \widehat{Q} \backslash\left\{q_{K}\right\}$,

$$
\begin{aligned}
\widehat{V}_{i}\left(q_{l}, \bar{s}^{l}\right)-p_{i}^{l} & \leq \widehat{V}_{i}\left(q_{j}, s^{j}\right)-p_{i}^{j} \\
& <\widehat{V}_{i}\left(q_{K}, s^{K}\right)-p_{i}^{K}<V_{i}\left(q_{k}, s^{k}\right)-p_{i}^{k}
\end{aligned}
$$

where the strict inequalities follow from (30).

We make three observations.

(i) If $q_{k} \in \widehat{Q}$ (or $=q_{K}$ ), then the first and last terms from (32) would yield

$$
\begin{array}{r}
\max _{s \in \mathcal{N} \mathcal{E}^{\left(V_{i}, V_{-i}\right)}\left(q_{k}\right)} V_{i}\left(q_{k}, s\right)+\varepsilon(\text { or }+\gamma)-p_{i}^{k}<V_{i}\left(q_{k}, s^{k}\right)-p_{i}^{k} \\
\Longrightarrow V_{i}\left(q_{k}, s^{k}\right)+\varepsilon(\text { or }+\gamma)-p_{i}^{k}<V_{i}\left(q_{k}, s^{k}\right)-p_{i}^{k},
\end{array}
$$

which is a contradiction. Thus, $q_{k} \notin \widehat{Q} \cup\left\{q_{K}\right\}$.

(ii) Because $q_{k} \notin \widehat{Q} \cup\left\{q_{K}\right\}$, it follows from Lemma B.3(ii) and (32) that $\widehat{V}_{i}\left(q_{K}, s^{K}\right)-p_{i}^{K}<\widehat{V}_{i}\left(q_{k}, s^{k}\right)-p_{i}^{K}$ implies that $f\left(\widehat{V}_{i}, V_{-i}\right) \neq q_{K}$.

(iii) Finally, since $\widehat{V}_{i}\left(q_{l}, \bar{s}^{l}\right)-p_{i}^{l}<\widehat{V}_{i}\left(q_{K}, s^{K}\right)-p_{i}^{K}$, it follows from Lemma B.3(i) that $f\left(\widehat{V}_{i}, V_{-i}\right) \neq q_{l}$ for any $q_{l} \in \widehat{Q} \backslash\left\{q_{K}\right\}$.

Based on these three observations, it follows that $f\left(\widehat{V}_{i}, V_{-i}\right)=q_{\widehat{k}} \notin \widehat{Q} \cup\left\{q_{K}\right\}$. However,

$$
0=\widehat{V}_{i}\left(q_{\widehat{k}}, s^{\widehat{k} *}\right)-V_{i}\left(q_{\widehat{k}}, s^{\widehat{k} *}\right)<\widehat{V}_{i}\left(q_{j}, s^{j}\right)-V_{i}\left(q_{j}, s^{j}\right)=\varepsilon,
$$

which violates Nash-MON. This contradiction means that Nash-MON is sufficient for $f$ to be truthful in a rich environment with ex post hidden actions.

Proof of Claim 1. Suppose that $q_{r} \geq_{i} q_{t}$. Then $q_{t} \neq q_{K}$. Let $s^{r} \in \mathcal{N} \mathcal{E}^{\left(V_{i}, V_{-i}\right)}\left(q_{r}\right)$ and $s^{t} \in \mathcal{N} \mathcal{E}^{\left(V_{i}, V_{-i}\right)}\left(q_{t}\right)$. If $q_{t} \notin \widehat{Q}$ then

$$
\widehat{V}_{i}\left(q_{t}, s^{t}\right)=\underbrace{V_{i}\left(q_{t}, s^{t}\right) \leq V_{i}\left(q_{r}, s^{r}\right)}_{\text {Consistency of } V_{i}} \leq V_{i}\left(q_{r}, s^{r}\right)+0(\text { or }+\gamma \text { or }+\varepsilon)
$$

where the first inequality follows from the consistency of $V_{i}$, and $(+\gamma)$ if $q_{r}=q_{K}$ or $(+\varepsilon)$ if $q_{r} \in \widehat{Q} \backslash\left\{q_{K}\right\}$. Consistency of $\widehat{V}_{i}$ thus follows.

Therefore, suppose that $q_{t} \in \widehat{Q}$. Three cases must be considered. 


\section{Case 1}

If $q_{r} \in \widehat{Q} \backslash\left\{q_{K}\right\}$ then

$$
\widehat{V}_{i}\left(q_{t}, s^{t}\right)=\underbrace{V_{i}\left(q_{t}, s^{t}\right)+\varepsilon \leq V_{i}\left(q_{r}, s^{r}\right)+\varepsilon}_{\text {Consistency of } V_{i}}=\widehat{V}_{i}\left(q_{r}, s^{r}\right)
$$

where the inequality follows from the consistency of $V_{i}$. Consistency of $\widehat{V}_{i}$ thus follows.

\section{Case 2}

If $q_{r}=q_{K} \geq_{i} q_{t}$ then, by the consistency of $V_{i}$ and $\gamma>\varepsilon$,

$$
\begin{aligned}
\widehat{V}_{i}\left(q_{r}, s^{r}\right) & =\max _{s^{K} \in \mathcal{N} \mathcal{E}^{\left(V_{i}, V_{-i}\right)}\left(q_{K}\right)} V_{i}\left(q_{K}, s^{K}\right)+\gamma \\
& >\max _{s^{K} \in \mathcal{N} \mathcal{E}^{\left(V_{i}, V_{-i}\right)}\left(q_{K}\right)} V_{i}\left(q_{K}, s^{K}\right)+\varepsilon \\
& \geq \max _{s^{t} \in \mathcal{N} \mathcal{E}^{\left(V_{i}, V_{-i}\right)}\left(q_{t}\right)} V_{i}\left(q_{t}, s^{t}\right)+\varepsilon=\widehat{V}\left(q_{t}, s^{t}\right) .
\end{aligned}
$$

where the weak inequality follows from the consistency of $V_{i}$. Consistency of $\widehat{V}_{i}$ thus follows in this case.

\section{Case 3}

If $q_{r} \notin \widehat{Q} \cup\left\{q_{K}\right\}$ then

$$
\max _{s^{r} \in \mathcal{N} \mathcal{E}^{\left(V_{i}, V_{-i}\right)}\left(q_{r}\right)} V_{i}\left(q_{r}, s^{r}\right) \neq V_{i}\left(q_{j}, s^{j}\right)=\max _{s^{t} \in \mathcal{N} \mathcal{E}^{\left(V_{i}, V_{-i}\right)}\left(q_{t}\right)} V_{i}\left(q_{t}, s^{t}\right) .
$$

This means that

$$
V_{i}\left(q_{j}, s^{j}\right)=\max _{s^{t} \in \mathcal{N} \mathcal{E}^{\left(V_{i}, V_{-i}\right)}\left(q_{t}\right)} V_{i}\left(q_{t}, s^{t}\right)<\max _{s^{r} \in \mathcal{N} \mathcal{E}^{\left(V_{i}, V_{-i}\right)}\left(q_{r}\right)} V_{i}\left(q_{r}, s^{r}\right) \neq V_{i}\left(q_{j}, s^{j}\right),
$$

\section{Consistency of $V_{i}$}

where strictness is necessary for $(\neq)$ to be true. As such, a small enough $\varepsilon>0$ can be selected to ensure that $\widehat{V}_{i}\left(q_{t}, s^{t}\right)=\max _{s^{t} \in \mathcal{N} \mathcal{E}^{\left(V_{i}, V_{-i}\right)}\left(q_{l}\right)} V_{i}\left(q_{t}, s^{t}\right)+\varepsilon<\max _{s^{r} \in \mathcal{N} \mathcal{E}^{\left(V_{i}, V_{-i}\right)}\left(q_{r}\right)} V_{i}\left(q_{r}, s^{r}\right)=\widehat{V}_{i}\left(q_{r}, s^{r}\right)$.

Consistency of $\widehat{V}_{i}$ thus follows. 


\section{References}

Aumann, R. J. (1976). Agreeing to disagree. The Annals of Statistics, 4(6), 1236-1239.

Bergemann, D., \& Morris, S. (2005). Robust mechanism design. Econometrica, 73(6), 1771-1813.

Bergemann, D., \& Morris, S. (2011). Robust implementation in general mechanisms. Games and Economic Behavior, 71(2), 261-281.

Bergemann, D., Morris, S., \& Tercieux, O. (2011). Rationalizable implementation. Journal of Economic Theory, 146(3), 1253-1274.

Bernheim, D. (1984). Rationalizable strategic behavior. Econometrica, 52(4), 1007-1028.

Bikhchandani, S., Chatterji, S., Lavi, R., Mu'alem, A., Nisan, N., \& Sen, A. (2006). Weak monotonicity characterizes deterministic dominant-strategy implementation. Econometrica, 74(4), 1109-1132.

Chung, K.-S., \& Ely, J. C. (2007). Foundations of dominant-strategy mechanisms. Review of Economic Studies, 74(2), 447-476.

Dasgupta, P., Hammond, P., \& Maskin, E. (1979). The implementation of social choice rules: Some general results on incentive compatibility. Review of Economic Studies, 46(2), 185-216.

Dasgupta, P., \& Maskin, E. (2000). Efficient auctions. Quarterly Journal of Economics, 115(2), 341-388.

Jackson, M. O., \& Palfrey, T. R. (2001). Voluntary implementation. Journal of Economic Theory, 98(1), 1-25.

Jehiel, P., Meyer-ter-Vehn, M., Moldovanu, B., \& Zame, W. R. (2006). The limits of ex post implementation. Econometrica, 74(3), 585-610.

Jehiel, P., \& Moldovanu, B. (2001). Efficient design with interdependent valuations. Econometrica, 69(5), 1237-1259.

Jehiel, P., Moldovanu, B., \& Stacchetti, E. (1999). Multidimensional mechanism design for auctions with externalities. Journal of Economic Theory, 85(2), 258-293.

Klemperer, P. (2002a). How (not) to run auctions: The European 3G telecom auctions. European Economic Review, 46(4-5), 829-845.

Klemperer, P. (2002b). What really matters in auction design. Journal of Economic Perspectives, 16(1), 169-189.

Krishna, V., \& Maenner, E. (2001). Convex potentials with an application to mechanism design. Econometrica, 69(4), 1113-1119.

Ma, C.-T., Moore, J., \& Turnbull, S. (1988). Stopping agents from "cheating". Journal of Economic Theory, 46(2), 355-372.

Maskin, E. (1999). Nash equilibrium and welfare optimality. Review of Economic Studies, 66(1), 23-38. 
McAfee, R. P., \& McMillan, J. (1988). Multidimensional incentive compatibility and mechanism design. Journal of Economic Theory, 46(2), 335-354.

McAfee, R. P., \& McMillan, J. (1996). Analyzing the airwaves auction. Journal of Economic Perspectives, 10(1), 159-175.

Milgrom, P. (2000). Putting auction theory to work: The simultaneous ascending auction. Journal of Political Economy, 108(2), 245-272.

Milgrom, P., \& Segal, I. (2002). Envelope theorems for arbitrary choice sets. Econometrica, 70(2), 583-601.

Myerson, R. B. (1981). Optimal auction design. Mathematics of Operations Research, 6(1), 58-73.

Pearce, D. G. (1984). Rationalizable strategic behavior and the problem of perfection. Econometrica, 52(4), 1029-1050.

Roberts, K. (1979). The characterization of implementable choice rules. In J.-J. Laffont (Ed.), Aggregation and revelation of preferences (pp. 321-345). Amsterdam: NorthHolland.

Rochet, J.-C. (1987). A necessary and sufficient condition for rationalizability in a quasi-linear context. Journal of Mathematical Economics, 16(2), 191-200.

Williams, S. R. (1999). A characterization of efficient, Bayesian incentive compatible mechanisms. Economic Theory, 14(1), 155-180.

Wilson, R. (1987). Game-theoretic approaches to trading processes. In T. Bewley (Ed.), Advances in economic theory: Fifth world congress (pp. 33-77). Cambridge: Cambridge University Press.

Wolitzky, A. (2016). Mechanism design with maxmin agents: Theory and an application to bilateral trade. Theoretical Economics, 11(3), 971-1004. 